\title{
On Certain Two Dimensional Integrals that Appear In Conformal Field Theory
}

\author{
Jeffrey S. Geronimo* \\ School of Mathematics \\ Georgia Institute of Technology \\ Atlanta, GA 30332-0160 \\ Henri Navelet \\ CEA- Saclay \\ Service de Physique Theorique \\ F-91191 Gif-sur-Yvette Cedex, France
}

November 4, 2018

\section{Introduction}

In the framework of perturbative QCD at large $Q^{2}$ and low $x$, the conformal (global) group of transformations in the transverse coordinate space plays a crucial role. Indeed Lipatov and his collaborators [1] have derived the master equation for the derivative of the gluon structure function with respect to $x$ by resuming the leading $\left(\alpha_{s}\left(\bar{Q}^{2}\right) \log (1 / x)\right)^{n}$ terms at fixed $\alpha_{s}\left(\bar{Q}^{2}\right)$. This derivative is a convolution in the transverse space of the gluon structure function times a conformal invariant kernel (BKKL kernel). All the relevant observables are then expressed as an expansion over the basis $E^{n, \nu}$ of the conformal eigenfunctions of this kernel. The integer $n$ is the conformal spin and $i \nu$ corresponds to a continuous imaginary scaling dimension. Among others, the elastic off mass shell gluon-gluon amplitude corresponding to the exchange of the bare QCD hard Pomeron $[1,2]$ and the conformal invariant triple

\footnotetext{
* Supported in part by a grant from the Fulbright Foundation and NSF.
} 
Pomeron vertex [3]. For this purpose one is led following [1] to consider the eigenfunctions in a mixed representation which is obtained by a Fourier transform in 2 dimensions of $E^{n, \nu}$. To be more specific, these eigenfunctions in the coordinate space are the product of a holomorphic times an antiholomorphic function. Recently, it has been shown [2] that the answer is a sum of two products of a holomorphic times an antiholomorphic form. This was accomplished by noticing that the Fourier transform is solution of a set of two linear differential equations one with respect to the complex variable and the other with respect to the complex conjugate variable. This structure of conformal blocks is already present in the computation of correlation functions in 2 dimensional conformal invariant quantum field theories [4]. Various theorems have been established for an integrand with integrable singularities at 0,1 and $\infty$. In reference [2] a similar result seems to apply, as shown by the existence in this case of 2 differential equations. In the first part of this paper we show that this is not a sheer coincidence but that similar theorems can apply for the bidimensional Fourier transform. As far as the triple conformal invariant Pomeron is concerned, specific analytic calculations have been done recently [3]. It turns out that this quantity is a peculiar case of a more general calculation involving a p-tuple conformally invariant integral in the complex space at a particular complex value of $z$ (resp $\bar{z})$. The triple Pomeron corresponds to the case $z=1$ and $p=3$. Our second part is the derivation of this very general result. The key point is to find the set of two linear differential equations with respect to $z$ and $\bar{z}$ of order $p+1$ which are obeyed by the p-tuple integral. The final answer will thus be a sum of conformal blocks, as expected, each block being the product of one of the $p+1$ solutions in $z$ of the differential equation times the corresponding one in $\bar{z}$, in order to preserve the single valuedness of the solution. In our study, the system of linear equations is the one obeyed by the hypergeometric function ${ }_{p+1} F_{p}(z)$. The case of degeneracy which is relevant for the calculation of the triple Pomeron vertex will be treated explicitly in a forthcoming paper.

\section{Bidimensional Fourier Transforms}

We begin this section with the following,

Lemma 1 For $0 \leq x \leq y \leq \frac{\pi}{2}$, let

$$
I_{1}=\int_{0}^{\frac{\pi}{2}}\left(\int_{0}^{y} e^{-R(\sin y-\sin x)} d x\right) d y .
$$

Then $\left|I_{1}\right| \leq C_{\epsilon} R^{\epsilon-1}$, for any $0<\epsilon \leq 1$. 
Proof. It follows from the mean value Theorem and Jordan's lemma that for $0 \leq x \leq$ $y \leq \frac{\pi}{2}, \sin y-\sin x \geq \frac{\pi}{2}\left(\frac{\pi}{2}-y\right)(y-x)$, consequently,

$$
\int_{0}^{y} e^{-R(\sin y-\sin x)} d x \leq \int_{0}^{y} e^{-R \frac{\pi}{2}\left(\frac{\pi}{2}-y\right)(y-x)} d x=\frac{\pi}{2 R} \frac{1-e^{-\frac{2 R}{\pi}\left(\frac{\pi}{2}-y\right) y}}{\left(\frac{\pi}{2}-y\right)} .
$$

Substituting the above result into $I_{1}$, then integrating by parts yields

$$
I_{1} \leq \int_{0}^{\frac{\pi}{2}} \ln \left(\frac{\pi}{2}-y\right) e^{-\frac{2 R}{\pi}\left(\frac{\pi}{2}-y\right) y}\left(\frac{\pi}{2}-2 y\right) d y .
$$

Since $\left|\left(\frac{\pi}{2}-y\right)^{\epsilon} \ln \left(\frac{\pi}{2}-y\right)\right| \leq K_{\epsilon}<\infty$ for $0 \leq y \leq \frac{\pi}{2}, \epsilon>0$ with $K_{\epsilon}$ monotonically decreasing we see that

$$
\begin{aligned}
I_{1} & \leq \frac{\pi}{2} K_{\epsilon} \int_{0}^{\frac{\pi}{2}}\left(\frac{\pi}{2}-y\right)^{-\epsilon} e^{-\frac{2 R}{\pi}\left(\frac{\pi}{2}-y\right) y} d y \\
& \leq \frac{\pi}{2} K_{\epsilon}\left(\frac{\pi}{4}\right)^{-\epsilon} \int_{0}^{\frac{\pi}{4}} e^{-\left(\frac{R}{2} y\right)} d y+\frac{\pi}{2} K_{\epsilon} \int_{\frac{\pi}{2}}^{\frac{\pi}{4}}\left(\frac{\pi}{2}-y\right)^{-\epsilon} e^{-\frac{2 R}{\pi}\left(\frac{\pi}{2}-y\right) y} d y .
\end{aligned}
$$

Thus extending the region of integration in the first integral to the full semi-axis then doing same thing to the second integral after the change of variables $y_{1}=\frac{\pi}{2}-y$ yields,

$$
I_{1} \leq \frac{\pi}{2}\left(\frac{\pi}{4}\right)^{-\epsilon} \frac{2 K_{\epsilon}}{R}+\frac{\pi}{2} K_{\epsilon}\left(\frac{2}{R}\right)^{1-\epsilon} \Gamma(1-\epsilon) .
$$

which gives the result.

With the above lemma we can now factorize certain double integrals. We will consider integrals of the form

$$
I=\iint_{\mathbb{C} \backslash[0, \infty)} f(t) \overline{g(t)} d^{2} t,
$$

where $\mathbb{C} \backslash[0, \infty)$ is the complex plane cut along the real axis from zero to infinity. We will make the following assumptions about $f$ and $g$.

i) I converges.

ii) $f(t)=\tilde{f}(t) e^{i q \cdot t}, g(t)=\tilde{g}(t) e^{i q \cdot t}, q \in \mathbb{R}$.

iii) $\tilde{f}(t)$ and $\tilde{g}(t)$ are analytic for $t \in \mathbb{C} \backslash[0, \infty)$. 
iv) $k_{f}=\left|\int_{0}^{\infty} f(x) d x\right|<\infty, k_{g}=\left|\int_{0}^{\infty} g(x) d x\right|<\infty$.

v) For $\epsilon$ small enough but positive $\lim _{R \rightarrow \infty} k_{\tilde{g}}(R) R^{\epsilon}=0, \lim _{R \rightarrow \infty} k_{\tilde{f}}(R) R^{\epsilon}=0$ and $\lim _{R \rightarrow \infty} k_{\tilde{g}}(R) k_{\tilde{f}}(R) R^{1+\epsilon}=0$, where $k_{\tilde{g}}(R)=\max _{\theta \in[0,2 \pi)}\left|\tilde{g}\left(R e^{i \theta}\right)\right|$ and $k_{\tilde{f}}(R)=\max _{\theta \in[0,2 \pi]}\left|\tilde{f}\left(R e^{i \theta}\right)\right|$. Finally

vi) $\lim _{r \rightarrow 0} k_{\tilde{f}}(r) r=0=\lim _{r \rightarrow 0} k_{\tilde{g}}(r) r$.

With the above assumptions $I=\lim _{R \rightarrow \infty} I_{R}$ where

$$
I_{R}=\iint_{D_{R} \backslash[0, R)} f(t) \overline{g(t)} d^{2} t
$$

with $D_{R}$ being the disk of radius $R$. We proceed to cut $D_{R} \backslash[0, \infty)$ into the components indicated in Figure 1 below. If we choose the point $z_{1}$ as the base point then Stoke's Theorem implies that

$$
I_{R}=\lim _{\Delta \rightarrow 0} \frac{i}{2} \int_{\partial D_{1}+\partial D_{2}} f_{1}(t) \overline{g(t)} \overline{d t}
$$

where $\partial D_{i}, i=1,2$ indicate the boundary of $D_{i}, i=1,2$ respectively, $f_{1}(t)=$ $\int_{\Gamma_{1, t}} f(y) d y, \Gamma_{1, t}$ being the path followed from the point $z_{1}$ to $t$ along the contour indicated on $\partial D_{1}$ or $\partial D_{2}$. Note that the contributions to $I_{R}$ along $\Gamma_{8541}$ on $\partial D_{2}$ cancels with the contribution of $\Gamma_{1458}$ on $\partial D_{1}$. The integral

$$
\begin{aligned}
\left|\int_{\Gamma_{21}} f_{1}(t) \overline{g(t)} \overline{d t}\right| & =\mid \int_{0}^{\pi / 2} d \phi \int_{\phi}^{\pi / 2} d \theta f\left(R e^{i \theta} \overline{g\left(R e^{i \phi}\right)} e^{i(\theta-\phi)} R^{2} \mid\right. \\
& \leq k_{\tilde{g}}(R) k_{\tilde{f}}(R) R^{2} \int_{0}^{\pi / 2} d \phi \int_{\phi}^{\pi / 2} d \theta e^{-q R(\sin \theta-\sin \phi)}
\end{aligned}
$$

which by Lemma 1 is bounded by

$$
\leq k_{\tilde{g}}(R) k_{\tilde{f}}(R) R^{1+\epsilon} .
$$

$\epsilon$ small but positive. Since

$$
\int_{\Gamma_{18}} f_{1}(t) \overline{g(t)} \overline{d t}=\int_{\pi / 2}^{3 \pi / 2} d \phi \int_{\pi / 2}^{\phi} d \theta f\left(R e^{i \theta}\right) \overline{g\left(R e^{i \phi}\right)} e^{i(\theta-\phi)} R^{2}
$$


An argument similar to the one above results in a bound similar to (1). The integral

$$
\begin{aligned}
& \left|\int_{\Gamma_{87}} f_{1}(t) \overline{g(t)} \overline{d t}\right|= \\
& \quad\left|\int_{\Gamma_{87}}\left(f_{7}+f_{6}-f_{7}+f_{3}-f_{6}+f_{2}-f_{3}+f_{1}-f_{2}\right) \overline{g(t)} \overline{d t}\right|
\end{aligned}
$$

and is bounded by

$$
\leq 2 k_{\tilde{g}}(R) k_{\tilde{f}}(R) R^{1+\epsilon}+2 k_{f} k_{\tilde{g}} R^{\epsilon}+r k_{\tilde{f}}(r) k_{\tilde{g}}(R) R^{\epsilon}
$$

The above inequalities also show that

$$
\begin{gathered}
\lim _{\Delta \rightarrow 0}\left|\int_{\Gamma_{63}} f_{1}(t) \overline{g(t)} d t\right|=0 \\
\left|\int_{\gamma_{32}} f_{1}(t) \overline{g(t)} \overline{d t}-\int_{\Gamma_{32}} f_{2} \overline{g(t)} \overline{d t}\right| \leq k_{g} k_{\tilde{f}} R^{\epsilon}
\end{gathered}
$$

and

$$
\lim _{\Delta \rightarrow 0}\left|\int_{\Gamma_{76}} f_{1}(t) \overline{g(t)} \overline{d t}-\left(\left(f_{2}-f_{3}\right)+\left(f_{6}-f_{7}\right)+f_{7}(t)\right) \overline{g(t)} \overline{d t}\right| \leq k_{g} k_{\tilde{f}} R_{+}^{\epsilon}
$$

This leads to,

Lemma 2 Suppose $f$ and $g$ satisfy i)-vi) then

$$
\begin{gathered}
\lim _{R \rightarrow \infty} \mid I_{R}-\lim _{\Delta \rightarrow 0}\left(\frac{i}{2} \int_{\Gamma_{76}}\left(\left(f_{2}-f_{3}\right)+\left(f_{6}-f_{7}\right)\right) \overline{g(t)} \overline{d t}\right. \\
\left.\left.+\frac{i}{2} \int_{\Gamma_{32}} f_{2}(t) \overline{g(t)} \overline{d t}+\frac{i}{2} \int_{\Gamma_{76}} f_{7}(t) \overline{g(t)} \overline{d t}\right)\right) \mid=0
\end{gathered}
$$

We now consider the integral

$$
I=\iint_{\hat{C}} t^{\gamma-1} \bar{t}^{\gamma-1} e^{i(\bar{q} \cdot t+q \cdot \bar{t})} d^{2} t
$$

where $\hat{C}$ is the complex plane cut so as to make $\ln t$ well defined. By rotating and scaling the coordinate system we may write

$$
I=q^{-\tilde{\gamma}} \bar{q}^{-\gamma} \hat{I}
$$


where

$$
I=\iint_{\mathbb{C} \backslash[0, \infty)} t^{\gamma-1} \overline{t^{\gamma}-1} e^{i(t+\bar{t})} d^{2} t .
$$

In the above integral the branch of the logarithm selected is such that $0 \leq \arg t<2 \pi$ and $\ln t$ is positive for $t>1$. With $t=r e^{i \theta}$ set

$$
f(t)=t^{\gamma-1} e^{i r \cos \theta-r \sin \theta}
$$

and

$$
g(t)=t^{\overline{\tilde{\gamma}}-1} e^{-i r \cos \theta+r \sin \theta}
$$

Thus we find

$$
\hat{I}=\int_{0}^{\infty} \int_{0}^{2 \pi} r^{\gamma+\tilde{\gamma}-1} e^{i(\gamma-\tilde{\gamma}) \theta} e^{2 r i \cos \theta} d r d \theta \quad q>0 .
$$

Since $\int_{0}^{2 \pi} e^{i(\gamma-\tilde{\gamma}) \theta} e^{2 i r \cos \theta} d \theta=O\left(\frac{1}{\sqrt{r}}\right)$ we see that $\hat{I}$ converges uniformly for $0<$ $\operatorname{Re}(\gamma+\tilde{\gamma})<1 / 2$ and thus defines an analytic function of $\gamma$ and $\tilde{\gamma}$ for the values of these variables restricted to this domain.

We now consider the integral

$$
I_{R}=\int_{D_{R} \backslash[0, R]} f(t) \overline{g(t)} d^{2} t
$$

with $0<\operatorname{Re} \gamma<1 / 2,0<\operatorname{Re} \tilde{\gamma}<1 / 2$ and $0<\operatorname{Re}(\gamma+\tilde{\gamma})<1 / 2$. If $f_{1}(t)=t^{\gamma-1}$ and $g_{1}(t)=t^{\bar{\gamma}-1}$ the conditions of Lemma 2 are satisfied and taking into account the increment in the phase due to the cut we find

$$
\begin{aligned}
\hat{I}= & \lim _{R \rightarrow \infty} I_{R}=\frac{1}{2}\left(1-e^{2 \pi i \gamma}\right) e^{-2 \pi i \tilde{\gamma}} \int_{0}^{\infty} x^{\gamma-1} e^{i x} d x \int_{0}^{\infty} y^{\tilde{\gamma}-1} e^{i y} d y \\
& +\frac{1}{2}\left(e^{2 \pi i(\gamma-\tilde{\gamma})}-1\right) I(\gamma, \tilde{\gamma}),
\end{aligned}
$$

where

$$
I(\gamma, \tilde{\gamma})=\int_{0}^{\infty} d v \int_{v}^{\infty} d u u^{\gamma-1} v^{\tilde{\gamma}-1} e^{i(u+v)} .
$$

With the first two integrals replaced by their representation [6, Formula (33), p. 12] in terms of gamma functions we find

$$
\hat{I}=\frac{1}{2}\left(1-e^{2 \pi i \gamma}\right) e^{-2 \pi i \tilde{\gamma}} e^{i(\gamma+\tilde{\gamma}) \pi / 2} \Gamma(\gamma) \Gamma(\tilde{\gamma})+\frac{1}{2}\left(e^{2 \pi i(\gamma-\tilde{\gamma})}-1\right) I_{1}
$$


The first term can be analytically extended to $\gamma, \tilde{\gamma} \neq 0,-1,-2, \ldots$. To solve the 2 nd term we break it up into three integrals over the following regions $R_{1}=\{(v, u), 0 \leq$ $v<1, v \leq u<1\}, R_{2}=\{(u, v), 0 \leq v<1,1 \leq u \leq \infty\}$ and $R_{3}=\{(u, v), 1 \leq$ $v<\infty, v \leq u<\infty\}$. It is easy to see that $I_{1}$ can be analytically extended to $\gamma \neq 0,-1,-2, \ldots, \gamma+\tilde{\gamma} \neq 0,-1,-2, \ldots$ and $I_{2}=\int_{0}^{1} v^{\tilde{\gamma}} e^{i v} d u \int_{1}^{\infty} u^{\gamma-1} e^{i u} d u$ can be extended to the region $\operatorname{Re} \gamma<1, \tilde{\gamma} \neq 0,-1,-2, \ldots$. For $\operatorname{Re} \gamma<1$,

$$
\begin{aligned}
\left|I_{3}\right| & =\left|\int_{1}^{\infty} v^{\tilde{\gamma}-1} e^{i v} \int_{v}^{\infty} u^{\gamma-1} e^{i u} d u d v\right| \\
& \leq C \int_{1}^{\infty} v^{\operatorname{Re}(\tilde{\gamma}+\gamma)-2} d v
\end{aligned}
$$

which converges uniformly for $\operatorname{Re}(\gamma+\tilde{\gamma})<1$. Consequently $I_{3}$ can be extended to $\operatorname{Re} \gamma<1, \operatorname{Re}(\gamma+\tilde{\gamma})<1$. This implies that $I$ can be extended toe the region $\operatorname{Re}(\gamma)<1, \operatorname{Re}(\gamma+\tilde{\gamma})<1, \gamma, \tilde{\gamma} \neq 0,-1,-2, \ldots$. For $\operatorname{Re} \gamma>0 I(\gamma, \tilde{\gamma})$ can be written as

$$
\begin{aligned}
I(\gamma, \tilde{\gamma})= & \int_{0}^{\infty} \int_{0}^{\infty} u^{\gamma-1} v^{\tilde{\gamma}-1} e^{i(u+v)} d u d v \\
& -\int_{0}^{\infty} \int_{0}^{v} u^{\gamma-1} v^{\tilde{\gamma}-1} e^{i q(u+v)} d u d v .
\end{aligned}
$$

which allows an analytic extension for $\operatorname{Re} \gamma>0,0<\operatorname{Re}(\gamma+\tilde{\gamma})<1$ since this implies that $\operatorname{Re} \tilde{\gamma}<1$. Consequently we have shown

Theorem 1 For $0<\operatorname{Re}(\gamma+\tilde{\gamma})<1, \gamma, \tilde{\gamma} \neq 0,-1,-2, \ldots, q \neq 0$

$$
\begin{aligned}
I= & \frac{i}{2}\left(1-e^{2 \pi i \gamma}\right) e^{-2 \pi i \tilde{\gamma}} q^{-\tilde{\gamma}} \bar{q}^{-\gamma} e^{i(\gamma+\tilde{\gamma}) \pi / 2} \Gamma(\gamma) \Gamma(\tilde{\gamma}) \\
& +\frac{i}{2}\left(e^{2 \pi i(\gamma-\tilde{\gamma})}-1\right) I(\gamma, \tilde{\gamma}) .
\end{aligned}
$$

In particular if $\gamma-\tilde{\gamma}=n, n$ an integer the integral becomes

$$
I=\sin (\pi \gamma) e^{i(\pi / 2) n} q^{-\tilde{\gamma}} \bar{q}^{-\gamma} \Gamma(\gamma) \Gamma(\gamma+n)
$$

The next integral we will consider arises in the context of the QCD Domain. The integral to be considered is

$$
I=\iint d^{2} z \frac{e^{i / 2(q \bar{z}+\bar{q} z)}}{\left(z^{2}-\frac{\rho^{2}}{4}\right)^{u+1 / 2}\left(\bar{z}^{2}-\frac{\bar{\rho}^{2}}{4}\right)^{\hat{u}+1 / 2}},
$$




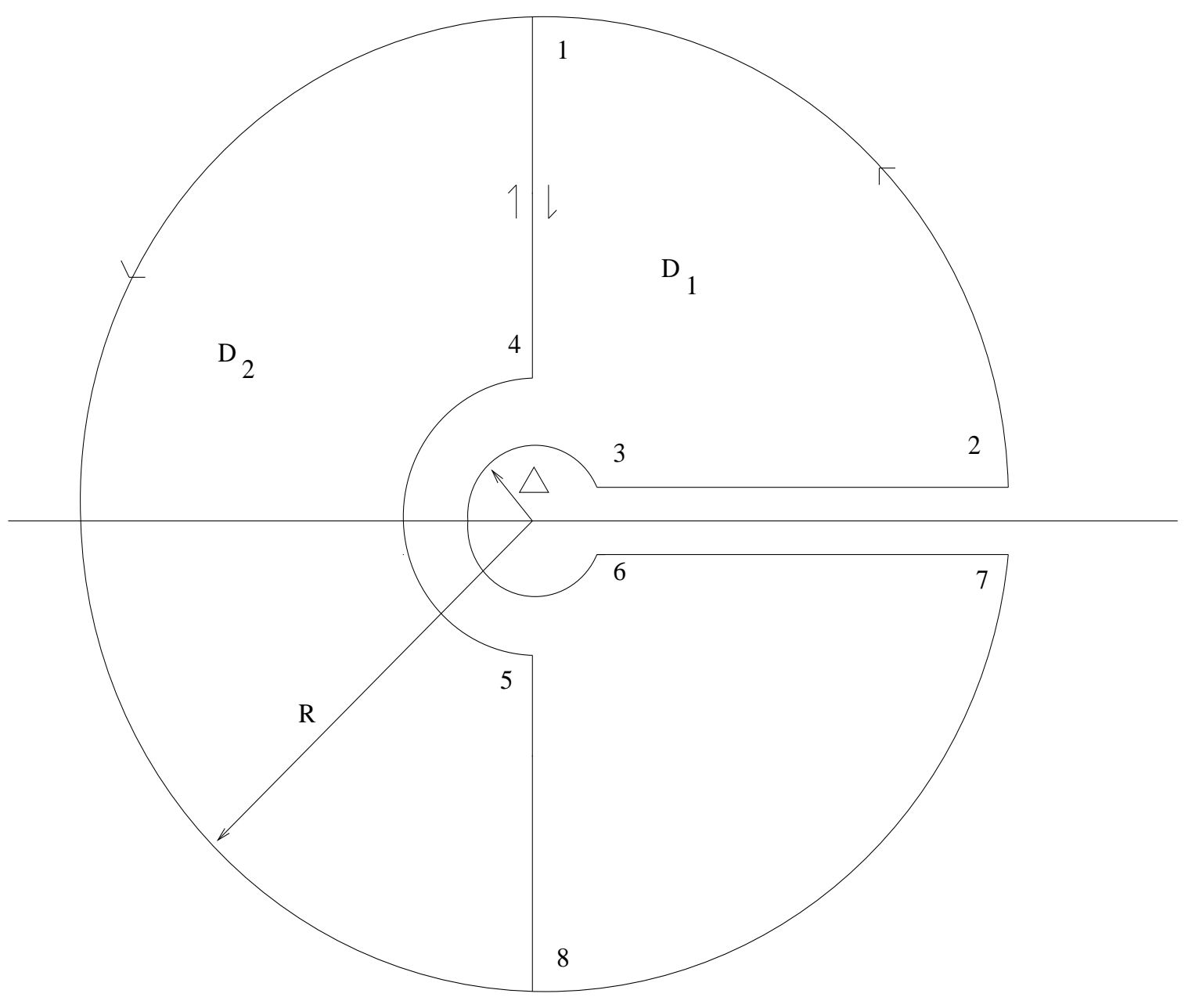

Figure 1: 
where $u=-v_{1}+i v_{2}, \hat{u}=v_{1}+i v_{2}$, and $\operatorname{Re}(q \bar{\rho}) \neq 0$. With an appropriate rotation and scaling the above integral becomes

$$
I=\frac{1}{\left(\frac{\rho^{2}}{4}\right)^{u}\left(\frac{\bar{\rho}^{2}}{4}\right)^{\hat{u}}} I_{1}
$$

with

$$
I_{1}=\iint_{\hat{C}} \frac{d^{2} z e^{\left(q_{1} \cdot \bar{z}+\bar{q}_{1} \cdot z\right)}}{\left(z^{2}-1\right)^{u+1 / 2}\left(\bar{z}^{2}-1\right)^{\hat{u}+1 / 2}}
$$

and $q_{1}=q \frac{\bar{\rho}}{4}$. Since $q_{1} \bar{z}$ comes in symmetrically with its complex conjugate we may assume that $\operatorname{Re} q_{1}>0$ and write $q_{1}=\hat{q}_{1} e^{i \phi}$. With this notation $\hat{\mathbb{C}}$ in the above integral is the complex plane cut from $\left[1, \infty e^{i \phi}\right)$ and from $\left(-\infty e^{-i \phi},-1\right]$. We use the branch of the logarithm so that the phases of $z-1$ and $z+1$ are equal to zero for $z>1$. Elementary methods including stationary phase shows that the above integral is convergent and defines an analytic function in the variables $u$ and $\hat{u}$ for $-1 / 4<\operatorname{Re}(u+\hat{u})<1$ and $\operatorname{Re} q_{1} \neq 0$ which is also continuous in $q_{1}$ in this region. We restrict $u$ and $\hat{u}$ to the region $-1 / 4<\operatorname{Re} u$, Re $\hat{u}<1 / 4$ and consider the integral

$$
I_{R}=\iint_{\hat{D}_{R}} \frac{d^{2} z e^{1 / 2\left(\bar{q}_{1} \cdot z+q_{1} \cdot \bar{z}\right)}}{\left(z^{2}-1\right)^{u+1 / 2}\left(\bar{z}^{2}-1\right)^{\hat{u}+1 / 2}}
$$

Using Stoke's Theorem we integrate over $\partial D_{1}$ and $\partial D_{2}$ given in Figure 2 with $\tilde{f}=$ $\left(z^{2}-1\right)^{-(u+1 / 2)}$ and $\tilde{g}=\left(z^{2}-1\right)^{\hat{\hat{u}}+1 / 2}$ to find,

$$
I_{R}=\frac{i}{2} \oint_{\partial D_{1}+\partial D_{2}} f_{1}(z) \overline{g(z)} d z .
$$

The conditions on $u$ and $\hat{u}$ are such that hypotheses $\mathrm{i}-\mathrm{iii}, \mathrm{v}$, and vi of Lemma 2 follow almost immediately. To verify iv) for the above functions we begin by showing that $\left|\int_{\Gamma_{23}} \overline{g(z)} d \bar{z}\right|<\infty$. Thus we need to show that $\lim _{R \rightarrow \infty}\left|\int_{0}^{r_{0}} \overline{g(z)} d \bar{z}\right|<\infty$ with $z=1+r e^{i \hat{\phi}}$, and with $r_{0}$ and $\hat{\phi}$ determined by the equation $1+r_{0} e^{i \hat{\phi}}=R e^{i \phi}$. The conditions on $r_{0}$ and $\hat{\phi}$ imply that $r_{0}=R(1+o(1))$ and $\hat{\phi}=\phi+O\left(\frac{1}{R}\right)$. Now

$$
\left|\int_{0}^{b} \bar{g} d \bar{z}\right|<C_{1} e^{\hat{q}_{1} b \sin (\hat{\phi}-\phi)} \int_{0}^{b} r^{-\operatorname{Re} \hat{u}-\frac{1}{2}} d r<\infty, \text { Re } \hat{u}<1 / 2,
$$

and using integration by parts,

$$
\left|\int_{b}^{r_{0}} \overline{g(z)} d \bar{z}\right|<C_{2} \frac{e^{\hat{q}_{1} \sin (\hat{\phi}-\phi)}}{|i \cos (\hat{\phi}-\phi)+\sin (\hat{\phi}-\phi)|} \frac{b^{-(2 \operatorname{Re} \hat{u}+1)}}{|q|}<\infty,-\frac{1}{2}<\operatorname{Re} \hat{u}
$$


for constants $C_{1}$ and $C_{2}$ independent of $R$. Applying the above reasoning to $f$ on $\Gamma_{45}$ shows that for $-1 / 4<\operatorname{Re} u, \operatorname{Re} \hat{u}<1 / 4$, iv is satisfied. Thus Lemma 2 (appropriately modified) shows that

$$
\begin{aligned}
I= & \lim _{R \rightarrow \infty} \frac{i}{2}\left[\int_{\Gamma_{54}}\left(f_{2}-f_{5}\right) \overline{g(z)} d \bar{z}+\int_{\Gamma_{54}} f_{5} \overline{g(z)} d \bar{z}+\int_{\Gamma_{32}} f_{2} \overline{g(z)} d \bar{z}\right] \\
& +\lim _{R \rightarrow \infty} \frac{i}{2}\left[\int_{\Gamma_{87}}\left(f_{10}-f_{7}\right) \overline{g(z)} d \bar{z}+\int_{\Gamma_{87}} f_{7} \overline{g(z)} d \bar{z}+\int_{\Gamma_{109}} f_{10} \overline{g(z)} d \bar{z}\right]
\end{aligned}
$$

From [7, p. 167 eq (6)] we see that

$$
\lim _{R \rightarrow \infty}\left(f_{5}-f_{2}\right)=\frac{i \pi \Gamma\left(\frac{1}{2}\right)\left(\frac{\bar{q}_{1}}{2}\right)^{u}}{\Gamma\left(u+\frac{1}{2}\right)} H_{-u}^{(1)}\left(\bar{q}_{1}\right),
$$

and

$$
\lim _{R \rightarrow \infty}\left(f_{10}-f_{7}\right)=\frac{i \pi \Gamma\left(\frac{1}{2}\right)\left(\frac{\bar{q}_{1}}{2}\right)^{u}}{\Gamma\left(u+\frac{1}{2}\right)} H_{-u}^{(2)}\left(\bar{q}_{1}\right) .
$$

Furthermore for $\hat{u}$ restricted to the region of interest the radius of small circular regions around \pm 1 may be taken to zero so that

$$
\begin{aligned}
\lim _{R \rightarrow \infty} \int_{\Gamma_{54}} \overline{g(z)} d \bar{z} & =-\int_{0}^{\infty} \frac{e^{i \hat{q}_{1} e^{i \phi}\left(1+r e^{-i \phi}\right)} e^{-i \phi}}{\left(r e^{-i \phi}\right)^{\hat{u}+\frac{1}{2}}\left(2+r e^{-i \phi}\right)^{\hat{u}+\frac{1}{2}}} d r \\
& =\frac{i \pi \Gamma\left(\frac{1}{2}\right)\left(\frac{q_{1}}{2}\right)^{\hat{u}}}{2 \Gamma\left(\frac{1}{2}-\hat{u}\right)} H_{\hat{u}}^{(1)}\left(q_{1}\right),
\end{aligned}
$$

and

$$
\lim _{R \rightarrow \infty} \int_{\Gamma_{87}} \overline{g(z)} d \bar{z}=\frac{i \pi \Gamma\left(\frac{1}{2}\right)\left(\frac{q_{1}}{2}\right)^{\hat{u}}}{2 \Gamma\left(\frac{1}{2}-\hat{u}\right)} H_{\hat{u}}^{(2)}\left(q_{1}\right) .
$$

Thus

$$
\begin{aligned}
I_{1}= & \frac{\pi \Gamma(1 / 2-\hat{u})}{4 \Gamma(1 / 2+u)}\left(\frac{q_{1}}{2}\right)^{\hat{u}}\left(\frac{\bar{q}_{1}}{2}\right)^{u} \\
& \times\left[H_{-u}^{1}\left(\bar{q}_{1}\right) H_{\hat{u}}^{1}\left(q_{1}\right)-H_{-u}^{2}\left(\bar{q}_{1}\right) H_{\hat{u}}^{2}\left(q_{1}\right)\right] \\
& +2 i \sin \pi(\hat{u}-u) \sin \left[\left(\pi-\bar{q}_{1}-q_{1}\right)(\hat{u}-u)\right] e^{i \phi(\hat{u}-u)} I\left(\hat{q}_{1}\right), q_{1} \neq 0
\end{aligned}
$$

where

$$
I\left(\hat{q}_{1}\right)=\int_{0}^{\infty} \int_{y}^{\infty} \frac{e^{i \hat{q}_{1}(r+y)}}{\left(r\left(2+r e^{i \phi}\right)\right)^{u+1 / 2}\left(y\left(2+y e^{-i \phi}\right)\right)^{\hat{u}+1 / 2}} d r d y .
$$


Using an argument similar to the one given above but more tedious it can be shown that $I\left(\hat{q}_{1}\right)$ has an analytic extension $-1 / 4<\operatorname{Re} u+\operatorname{Re} \hat{u}<1, u, \hat{u} \neq 1 / 2+n, n=$ $0,1, \ldots$ Thus we have shown

Theorem 2 For $u=-v_{1}+i v_{2}, \hat{u}=v_{1}+i v_{2}, u, \hat{u} \neq 1 / 2+n, n=0,1, \ldots, I_{1}$ is given by equation (2). In particular if $v_{1}=n / 2, v_{2} \neq 0$ then

$$
\begin{aligned}
I_{1}= & \frac{\pi \Gamma(1 / 2-\hat{u})}{4 \Gamma(1 / 2+u)}\left(\frac{q_{1}}{2}\right)^{\hat{u}}\left(\frac{\bar{q}_{1}}{2}\right)^{u} \\
& {\left[H_{-u}^{1}\left(\bar{q}_{1}\right) H_{\hat{u}}^{1}\left(q_{1}\right)-H_{-u}^{2}\left(\bar{q}_{1}\right) H_{\hat{u}}^{2}\left(q_{1}\right)\right] \quad q_{1} \neq 0 . }
\end{aligned}
$$

\section{A p-uple conformal integral and applications}

We now consider the integral

$$
\begin{aligned}
& I_{p+1}\left(a_{0}, a_{i}, b_{i} ; \tilde{a}_{0}, \tilde{a}_{i}, \tilde{b}_{i} ; z, \bar{z}\right) \\
= & \left(\frac{1}{2 i}\right)^{p} \int \prod_{i=1}^{p} d^{2} z_{i}\left(z_{i}\right)^{a_{i}-1}\left(\bar{z}_{i}\right)^{\tilde{a}_{i}-1}\left(1-z_{i}\right)^{b_{i}-a_{i}-1}\left(1-\bar{z}_{i}\right)^{\tilde{b}_{i}-\tilde{a}_{i}-1} \\
& \left(1-\left(\prod_{i=1}^{p} z_{i}\right) z\right)^{-a_{0}}\left(1-\left(\prod_{i=1}^{p} \bar{z}_{i}\right) \bar{z}\right)^{-\tilde{a}_{0}}
\end{aligned}
$$

We will analyze the case when $a_{i}, \tilde{a}_{i}, b_{i}$ and $\tilde{b}_{i}$ obey the following conditions:

Condition C. For $i=0 \ldots, p$ and $j=1 \ldots, p$,
a) $b_{j}, \tilde{b}_{j}, a_{i}, \tilde{a}_{i} \notin \mathbf{Z}$
b) $a_{i}-a_{j}, \tilde{a}_{i}-\tilde{a}_{j}, b_{i \neq 0}-b_{j}, \tilde{b}_{i \neq 0}-\tilde{b}_{j}, i \neq j \notin \mathbf{Z}$.
c) $a_{i}-b_{j}, \tilde{a}_{i}-\tilde{b}_{j} \notin \mathbf{Z}$
d) $a_{i}-\tilde{a}_{i}, b_{j}-\tilde{b}_{j} \in \mathbf{Z}$. 


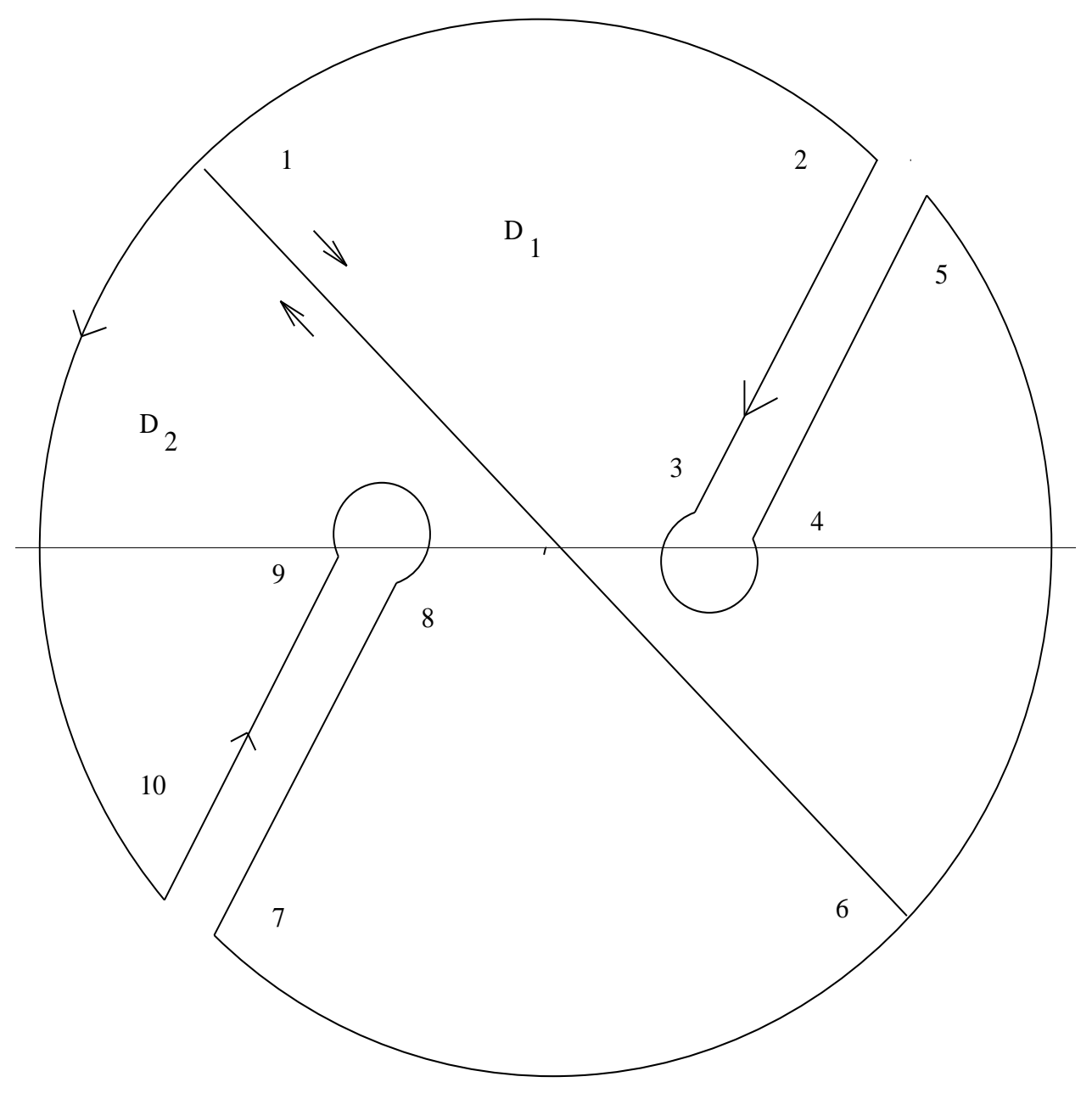

Figure 2: 
With $a^{j}=\left(a_{1}, \ldots, a_{j}\right), b^{j}=\left(b_{1}, \ldots, b_{j}\right), \tilde{a}^{j}=\left(\tilde{a}_{1}, \ldots, \tilde{a}_{j}\right), \tilde{b}^{j}=\left(\tilde{b}_{1}, \ldots, \tilde{b}_{j}\right)$, and $I_{1}\left(a_{0}, \tilde{a}_{0}, z, \bar{z}\right)=(1-z)^{-a_{0}}(1-\bar{z})^{-\tilde{a}_{0}}$ the above integral can be recast as the iterated integral

$$
\begin{aligned}
& I_{p+1}\left(a_{0}, a^{p}, b^{p} ; \tilde{a}_{0}, \tilde{a}^{p}, \tilde{b}^{p} ; z, \bar{z}\right) \\
&=\left(\frac{1}{2 i}\right) \quad \int d^{2} z_{p}\left(z_{p}\right)^{a_{p}-1}\left(1-z_{p}\right)^{b_{p}-a_{p}-1}\left(\bar{z}_{p}\right)^{\tilde{a}_{p}-1}\left(1-\bar{z}_{p}\right)^{\tilde{b}_{p}-\tilde{a}_{p}-1} \\
& \\
& I_{p}\left(a_{0}, a^{p-1}, b^{p-1} ; \tilde{a}_{0}, \tilde{a}^{p-1}, \tilde{b}^{p-1} ; z z_{p}, \bar{z} \bar{z}_{p}\right),
\end{aligned}
$$

The computation of this integral has already been done for $p=0$ and $p=1$ by various authors [4] in the case when $a_{i}=\tilde{a}_{i}, b_{i}=\tilde{b}_{i}$ and $a_{0}=\tilde{a}_{0}$. The result is a sum of a product of hypergeometric functions of $z$ with its antiholomorphic part of argument $\bar{z}$ exhibiting the conformal block structure. Lipatov [1] has given the general form for $p=1$ without the restrictions on the $\tilde{a}_{i}, \tilde{a}_{0}, \tilde{b}_{i}$.

We propose to give the exact analytic structure of $I_{p+1}$ for any positive integer value of $p$.

The key point of the calculation is to prove that the $I_{p+1}$ obey two linear differential equations of order $p+1$ namely

$$
O_{z}^{p+1}\left(a_{0}, a^{p}, b^{p}\right) I_{p+1}\left(a_{0}, a^{p}, b^{p} ; \tilde{a}_{0}, \tilde{a}^{p}, \tilde{b}^{p} ; z, \bar{z}\right) \equiv 0
$$

and

$$
O_{\bar{z}}^{p+1}\left(\tilde{a}_{0}, \tilde{a}^{p}, \tilde{b}^{p}\right) I_{p+1}\left(a_{0}, a^{p}, b^{p} ; \tilde{a}_{0}, \tilde{a}^{p}, \tilde{b}^{p} ; z, \bar{z}\right) \equiv 0
$$

where $O_{z}^{p+1}\left(\right.$ resp. $\left.O_{\bar{z}}^{p+1}\right)$ is the differential operator of order $p+1$ defining the hypergeometric function ${ }_{p+1} F_{p}\left(a_{0}, a^{p}, b^{p}, z\right)\left((\operatorname{resp} .)_{p+1} F_{p}\left(\tilde{a}_{0}, \tilde{a}^{p}, \tilde{b}^{p}, \bar{z}\right)\right)$ namely

$$
O_{z}^{p+1} \equiv \frac{\partial}{\partial z} \prod_{i=1}^{p}\left(z \frac{\partial}{\partial z}+b_{i}-1\right)-\prod_{i=0}^{p}\left(z \frac{\partial}{\partial z}+a_{i}\right)
$$

resp.

$$
O_{\bar{z}}^{p+1} \equiv \frac{\partial}{\partial \bar{z}} \prod_{i=1}^{p}\left(\bar{z} \frac{\partial}{\partial \bar{z}}+\tilde{b}_{i}-1\right)-\prod_{i=0}^{p}\left(\bar{z} \frac{\partial}{\partial \bar{z}}+\tilde{a}_{i}\right)
$$


The general solution of this system of differential equation reads

$$
I_{p+1}(z, \bar{z})=\sum_{i, j=0}^{p} \lambda_{i j}^{(p+1)} u_{i}^{p+1}(z) \tilde{u}_{j}^{p+1}
$$

where $u_{i}^{p+1}\left(a_{0}, a^{p}, b^{p}, z\right)\left(\operatorname{resp} . \tilde{u}_{j}^{p+1}\left(\tilde{a}_{0}, \tilde{a}^{p}, \tilde{b}^{p}, \bar{z}\right)\right)$ is any of the $p+1$ independent solutions of the differential equation

$$
O_{z}^{p+1} \varphi_{j}(z)=0 \quad j=0, . ., p
$$

namely

$$
u_{0}^{p+1}\left(a_{0}, a^{p}, b^{p}, z\right)={ }_{p+1} F_{p}\left(a_{0}, a^{p}, b^{p}, z\right),
$$

and

$$
\begin{aligned}
& u_{j}^{p+1}\left(a_{0}, a^{p}, b^{p}, z\right) \\
= & z^{1-b_{j}}{ }_{p+1} F_{p}\left(a_{0}-b_{j}+1, a_{i}-b_{j}+1,1+b_{i}-b_{j}, 2-b_{j} ; z\right)
\end{aligned}
$$

Since the solution we are looking for, has to be monovalued in $z \bar{z}$ the $\lambda_{i j}{ }^{\prime}$ s have to be diagonal provided none of the difference $b_{i}-b_{j}$ is an integer. Thus the general solution reduces to

$$
\begin{aligned}
& I_{p+1}\left(a_{0}, a^{p}, b^{p} ; \tilde{a}_{0}, \tilde{a}^{p}, \tilde{b}^{p} ; z, \bar{z}\right) \\
= & \sum_{j=0}^{p} \lambda_{j}^{p+1}\left(a_{0}, a^{p}, b^{p} ; \tilde{a}_{0}, \tilde{a}^{p}, \tilde{b}^{p}\right) u_{j}^{p+1}\left(a_{0}, a^{p}, b^{p} ; z\right) \widetilde{u}_{j}^{p+1}\left(\tilde{a}_{0}, \tilde{a}^{p}, \tilde{b}^{p} ; \bar{z}\right)
\end{aligned}
$$

which exhibits a conformal block structure.

The $p+1$ unknown constants $\lambda_{j}\left(a_{0}, a^{p}, b^{p}, \tilde{a}_{0}, \tilde{a}^{p}, \tilde{b}^{p}\right)$ are readily obtained by identifying the different behaviour of the solution $I_{p+1}$ near $z=0$.

At this stage we consider a generalized Euler function ([4]),

$$
B_{\alpha, \beta ; \tilde{\alpha}, \tilde{\beta}}=\frac{1}{2 i} \int d^{2} t t^{\alpha-1}(1-t)^{\beta-1}(\bar{t})^{\tilde{\alpha}-1}(1-\bar{t})^{\tilde{\beta}-1}
$$


which is defined and analytic for $\operatorname{Re}(\alpha+\tilde{\alpha})>0, \operatorname{Re}(\beta+\tilde{\beta})>0$, and $\operatorname{Re}(\alpha+\tilde{\alpha}+\beta+\tilde{\beta})<$ 2 . Using arguments similar to those given in the previous section the above integral can be evaluated so that for $\alpha-\tilde{\alpha}, \beta-\tilde{\beta}$ integer,

$$
B_{\alpha, \beta ; \tilde{\alpha}, \tilde{\beta}}=\frac{\Gamma(\alpha) \Gamma(\beta)}{\Gamma(\alpha+\beta)} \frac{\Gamma(\tilde{\alpha}) \Gamma(\tilde{\beta})}{\Gamma(\tilde{\alpha}+\tilde{\beta})} \frac{\sin \pi \tilde{\alpha} \sin \pi \tilde{\beta}}{\sin \pi(\tilde{\alpha}+\tilde{\beta})} .
$$

Note that since $\alpha-\tilde{\alpha}$ and $\beta-\tilde{\beta}$ are integer,

$$
\frac{\sin \pi \tilde{\alpha} \sin \pi \tilde{\beta}}{\sin \pi(\tilde{\alpha}+\tilde{\beta})} \equiv \frac{\sin \pi \alpha \sin \pi \beta}{\sin \pi(\alpha+\beta)}
$$

To find $\lambda_{0}^{p+1}$, set $z=0$ in (4) in which case the integral is readily obtained since it factorizes.

Thus,

$$
\lambda_{0}^{p+1}\left(a_{0}, a^{p}, b^{p}, \tilde{a}_{0}, \tilde{a}^{p}, \tilde{b}^{p}\right)=\prod_{i=1}^{p} B_{a_{i}, b_{i}-a_{i} ; \tilde{a}_{i}, \tilde{b}_{i}-\tilde{a}_{i}}
$$

which is $a_{0}, \tilde{a}_{0}$ independent.

To find $\lambda_{j}^{p+1}$, set $z_{j}=\left[\prod_{k=1, k \neq j}^{p} z_{k} z\right]^{-1} t$ and observe that

$$
\begin{aligned}
& d z_{j}\left(z_{j}\right)^{a_{j}-1}\left(1-z_{j}\right)^{b_{j}-a_{j}-1}\left(1-\left(\prod_{i=1}^{p} z_{i}\right) z\right)^{-a_{0}} \\
= & z^{1-b_{j}}(d t) t^{a_{j}-1}(1-t)^{-a_{0}}\left(\left(\prod_{k=1, k \neq j}^{p} z_{k}\right) z-t\right)^{b_{j}-a_{j}-1} \prod_{k=1, k \neq j}^{p}\left(z_{k}\right)^{1-b_{j}} .
\end{aligned}
$$

In a neighborhood of $z=0$ we find

$$
\begin{aligned}
I_{p+1} \sim & z^{1-b_{j}}(\bar{z})^{1-\tilde{b}_{j}}\left(\frac{1}{2 i}\right)^{p} \int d^{2} t t^{b_{j}-2}(1-t)^{-a_{0}}{\tilde{\tilde{t}^{j}}}^{-2}(1-\bar{t})^{-\widetilde{a}_{0}} \\
& \prod_{k=1, k \neq j}^{p} d^{2} z_{k}\left(z_{k}\right)^{a_{k}-b_{j}}\left(1-z_{k}\right)^{b_{k}-a_{k}-1}\left(\bar{z}_{k}\right)^{\widetilde{a}_{k}-\widetilde{b}_{j}} \\
& \left(1-\bar{z}_{k}\right)^{\widetilde{b}_{k}-\widetilde{a}_{k}-1}(-1)^{b_{j}-\tilde{b}_{j}-a_{j}+\tilde{a}_{j}}
\end{aligned}
$$


Thus,

$$
\begin{aligned}
\lambda_{j}^{p+1}\left(a_{0}, a^{p}, b^{p}, \widetilde{a}_{0}, \widetilde{a}^{p}, \widetilde{b}^{p}\right)= & (-1)^{b_{j}-\widetilde{b}_{j}-a_{j}+\widetilde{a}_{j}} B_{b_{j}-1,-a_{0}+1 ; \tilde{b}_{j}-1,-\widetilde{a}_{0}+1} \times \\
& \times \prod_{i=1, i \neq j}^{p} B_{a_{i}-b_{j}+1, b_{i}-a_{i} ; \widetilde{a}_{i}-\widetilde{b}_{j}+1, \widetilde{b}_{i}-\widetilde{a}_{i}}
\end{aligned}
$$

We now give some lemmas which will help prove that $I_{p+1}$ has the form indicated above.

Define,

$$
H_{j}^{p}\left(a_{0}, a^{p}, b^{p}, z, z_{p}\right)=\left(z_{p}\right)^{a_{p}-1}\left(1-z_{p}\right)^{b_{p}-a_{p}-1} u_{j}^{p}\left(a_{0}, a^{p-1}, b^{p-1}, z z_{p}\right),
$$

and

$$
K_{j}^{p}\left(a_{0}, a^{p}, b^{p}, z, z_{p}\right)=\left(z_{p}\right)^{a_{p}-1}\left(1-z_{p}\right)^{b_{p}-a_{p}+1} u_{j}^{p}\left(a_{0}, a^{p-1}, b^{p-1}, z z_{p}\right) .
$$

Lemma 3 Suppose condition $\mathbf{C}$ is satisfied. Then,

$$
O_{z}^{p+1} H_{0}^{(p)}\left(a_{0}, a^{p}, b^{p}, z, z_{p}\right)=-\prod_{i=0}^{p-1}\left(a_{i}\right) \frac{\partial}{\partial z_{p}} K_{0}^{p}\left(a_{0}+1, a^{p}+1, b^{p}, z, z_{p}\right)
$$

and for $j=1 \ldots p-1$,

$$
\begin{aligned}
& O_{z}^{p+1} H_{j}^{(p)}\left(a_{0}, a^{p}, b^{p}, z, z_{p}\right) \\
= & -\prod_{i=0}^{p-1}\left(a_{i}-b_{j}+1\right) \frac{\partial}{\partial z_{p}} K_{j}^{p}\left(a_{0}+1, a^{p}+1, b^{p}, z, z_{p}\right)
\end{aligned}
$$

Proof. Since

$$
\begin{gathered}
\left(z \frac{\partial}{\partial z}+a_{i}\right)_{p+1} F_{p}\left(a_{i}, b_{i}, z\right)=a_{i p+1} F_{p}\left(a_{i}+1, b_{i}, z\right) \\
\left(z \frac{\partial}{\partial z}+b_{i}-1\right)_{p+1} F_{p}\left(a_{i}, b_{i}, z\right)=\left(b_{i}-1\right)_{p+1} F_{p}\left(a_{i}, b_{i}-1, z\right),
\end{gathered}
$$


and

$$
\frac{\partial}{\partial z}{ }_{p+1} F_{p}\left(a_{i}, b_{i}, z\right)=a_{0} \prod_{k=1}^{p} \frac{a_{k}}{b_{k}}{ }_{p+1} F_{p}\left(a_{i}+1, b_{i}+1, z\right),
$$

equation (12) now follows after some algebra. Equation (13) similarly follows from the identities,

$$
\begin{gathered}
\left(z \frac{\partial}{\partial z}+a_{i}\right) z_{p+1}^{1-b_{j}} F_{p}\left(a_{i}-b_{j}+1,1+b_{i}-b_{j}, 2-b_{j} z\right) \\
=\left(a_{i}-b_{j}+1\right) z^{1-b_{j}}{ }_{p+1} F_{p}\left(a_{i}-b_{j}+2,1+b_{i}-b_{j}, 2-b_{j}, z\right) \\
\quad\left(z \frac{\partial}{\partial z}+b_{i}-1\right) z_{p+1}^{1-b_{j}} F_{p}\left(a_{i}-b_{j}+1,1+b_{i}-b_{j}, 2-b_{j}, z\right) \\
=\left(b_{i}-b_{j}\right) z_{p+1}^{1-b_{j}} F_{p}\left(a_{i}-b_{j}+1, b_{i}-b_{j}, 2-b_{j} z\right), \\
\left(z \frac{\partial}{\partial z}+b_{j}-1\right) z_{p+1}^{1-b_{j}} F_{p}\left(\left(a_{i}-b_{j}+1,1+b_{i}-b_{j}, 2-b_{j}, z\right)\right. \\
=\left(\frac{\left(a_{0}-b_{j}+1\right) \prod_{k=1}^{p}\left(a_{i}-b_{j}+1\right)}{\left(2-b_{j}\right) \prod_{k=1}^{p}\left(b_{i}-b_{j}+1\right)}\right) z_{p+1}^{2-b_{j}} F_{p}\left(a_{i}-b_{j}+2, b_{i}-b_{j}+2,3-b_{j} z\right),
\end{gathered}
$$

and

$$
\begin{aligned}
& \frac{\partial}{\partial z} z_{p+1}^{1-b_{j}} F_{p}\left(a_{i}-b_{j}+1, b_{i}-b_{j}+1,2-b_{j}, z\right) \\
= & \left(1-b_{j}\right) z_{p+1}^{-b_{j}} F_{p}\left(a_{i}-b_{j}+1, b_{i}-b_{j}+1,1-b_{j} z\right),
\end{aligned}
$$

Let $\hat{I}_{p+1}$ be given for $0<|z|<1$ by equations (8), (10), and (11) where we assume that $a^{p}$ and $b^{p}$ satisfy condition $C$. Since for $0<|z|<1$ the hypergeometric functions in $\hat{I}_{p+1}$ are defined by their series representation we seek a continuation of $\hat{I}_{p+1}$ to $|z|>1$. It follows from the Mellin-Barnes representation for ${ }_{p+1} F_{p}\left(a_{0}, a^{p}, b^{p}, z\right)$ (Luke [10] p. 149) that for $0<\arg z<2 \pi$

$$
\begin{aligned}
{ }_{p+1} F_{p}\left(a_{0}, a^{p}, b^{p}, z\right)= & \frac{\prod_{i=1}^{p} \Gamma\left(b_{i}\right)}{\prod_{i=0}^{p} \Gamma\left(a_{i}\right)} \sum_{j=0}^{p} \Gamma\left(a_{j}\right) \frac{\prod_{\substack{k=0 \\
k \neq j}}^{p} \Gamma\left(a_{k}-a_{j}\right)}{\prod_{k=1}^{p} \Gamma\left(b_{k}-a_{j}\right)}\left(\frac{e^{i \pi}}{z}\right)^{a_{j}} \\
& \times{ }_{p+1} F_{p}\left(a_{j}, 1+a_{j}-b_{i}, 1+a_{j}-a_{i}, \frac{1}{z}\right)
\end{aligned}
$$

The following Lemma will be useful it what follows: 
Lemma 4 Suppose the points $x_{i}$ and $y_{j}, i=0 \ldots p, j=1, \ldots p$ are distinct and none are equal to one. Then,

$$
\begin{aligned}
B_{n, k}= & \prod_{i=1}^{p} \frac{1-x_{i}}{1-y_{i}} \\
& +\sum_{j=1}^{p} \frac{\left(1-x_{n}\right)\left(1-x_{k}\right)\left(x_{0}-y_{j}\right)\left(y_{j}-x_{j}\right)}{\left(1-y_{j}\right)\left(1-x_{0}\right)\left(y_{j}-x_{n}\right)\left(y_{j}-x_{k}\right)} \prod_{\substack{i=1 \\
i \neq j}}^{p} \frac{\left(x_{i}-y_{j}\right)}{\left(y_{i}-y_{j}\right)} \\
= & \frac{u_{n} \prod_{\substack{i \neq 0 \\
i \neq j}}^{p}\left(u_{j}-u_{i}\right)}{u_{0} \prod_{i=1}^{p}\left(u_{j}-z_{i}\right)} \delta_{n, j}
\end{aligned}
$$

where $z_{k}=y_{k}-1$ and $u_{i}=x_{i}-1$.

Proof. $B_{n, k}$ is equal to

$$
B_{n, k}=\prod_{i=1}^{p} \frac{u_{i}}{z_{i}}+\sum_{j=1}^{p} \frac{u_{n} u_{k}\left(u_{0}-z_{j}\right)\left(z_{j}-u_{j}\right)}{z_{j} u_{0}\left(z_{j}-u_{n}\right)\left(z_{j}-u_{k}\right)} \prod_{\substack{i=1 \\ i \neq j}}^{p} \frac{\left(u_{i}-z_{j}\right)}{\left(z_{i}-z_{j}\right)}
$$

Write $B_{n, k} \prod_{1}^{p} \frac{z_{i}}{u_{i}}=S_{n, k}$ where,

$$
S_{n, k}=1-\lambda \sum_{j=1}^{p} \frac{\left(z_{j}-u_{0}\right)\left(z_{j}-u_{j}\right)}{z_{j}\left(z_{j}-u_{n}\right)\left(z_{j}-u_{k}\right)} \prod_{\substack{i=1 \\ i \neq j}}^{p} \frac{\left(u_{i}-z_{j}\right)}{\left(z_{i}-z_{j}\right)},
$$

and $\lambda=\frac{u_{k} u_{n}}{u_{0}} \prod_{i=1}^{p} \frac{z_{i}}{u_{i}}$. The function

$$
f(z)=\lambda \frac{\left(z-u_{0}\right)}{z\left(z-u_{n}\right)\left(z-u_{k}\right)} \prod_{i=1}^{p} \frac{\left(z-u_{i}\right)}{\left(z-z_{i}\right)}
$$

behaves as $z^{-2}$ for large $\mathrm{z}$, and by hypothesis has simple poles at $z=z_{j}$ and $z=0$. If $n=k$ then there is one extra simple pole at $z=u_{k}$. Cauchy's theorem says the sum of the residues is zero and the evaluation of the residues gives the result.

With $s\left(a_{i}\right)=\sin \pi a_{i}$ we now prove,

Lemma 5 Suppose $a_{0}, \tilde{a}_{0}, a^{p}, \tilde{a}^{p}, \tilde{b}^{p}$ and $b^{p}$ satisfy condition $\mathbf{C}$ then 


$$
\begin{aligned}
& \hat{I}_{p+1}(z, \bar{z})=\sum_{j=0}^{p} J_{j j} e^{i \pi\left(a_{j}-\tilde{a}_{j}\right)} z^{-a_{j}} \bar{z}^{-\tilde{a}_{j}}{ }_{p+1} F_{p}\left(a_{j}, a_{j}-b_{i}+1,1+a_{j}-a_{l \neq j}, \frac{1}{z}\right) \\
& \times_{p+1} F_{p}\left(\tilde{a}_{j}, \tilde{a}_{j}-\tilde{b}_{i}+1,1+\tilde{a}_{j}-\tilde{a}_{l \neq j}, \frac{1}{\bar{z}}\right)
\end{aligned}
$$

where $J_{j j}$ is given by,

$$
J_{j j}=\frac{s\left(\tilde{b}_{j}-\tilde{a}_{j}\right)}{s\left(\tilde{a}_{0}-\tilde{a}_{j}\right)} B_{a_{j}, a_{0}-a_{j}, \tilde{a}_{j}, \tilde{a}_{0}-a_{j}} \prod_{\substack{i=0 \\ i \neq j}}^{p} B_{b_{i}-a_{i}, a_{i}-a_{j}, \tilde{b}_{i}-\tilde{a}_{i}, \tilde{a}_{i}-\tilde{a}_{j}}, j \neq 0
$$

and

$$
J_{00}=\prod_{i=1}^{p} B_{b_{i}-a_{i}, a_{i}-a_{0}, \tilde{b}_{i}-\tilde{a}_{i}, \tilde{a}_{i}-\tilde{a}_{0}}
$$

Proof. From equation (14) it follows that

$$
\begin{aligned}
& \lambda_{0}^{p+1}{ }_{p+1} F_{p}\left(a_{0}, a^{p}, b^{p}, z\right)_{p+1} F_{p}\left(\tilde{a}_{0}, \tilde{a}^{p}, \tilde{b}^{p} ; \bar{z}\right)=\prod_{i=1}^{p} \frac{s\left(\tilde{a}_{i}\right) s\left(\tilde{b}_{i}-\tilde{a}_{i}\right)}{s\left(\tilde{b}_{i}\right)} \\
& \quad \times\left(\sum_{j=0}^{p} c_{0 j}^{p}\left(\frac{e^{i \pi}}{z}\right)^{a_{j}}{ }_{p+1} F_{p}\left(a_{j}, 1+a_{j}-b_{i}, 1+a_{j}-a_{l \neq j} ; \frac{1}{z}\right)\right) \\
& \quad \times\left(\sum_{j=0}^{p} \tilde{c}_{0 j}^{p}\left(\frac{e^{-i \pi}}{\bar{z}}\right)^{\tilde{a}_{j}}{ }_{p+1} F_{p}\left(\tilde{a}_{j}, 1+\tilde{a}_{j}-\tilde{b}_{i}, 1+\tilde{a}_{j}-\tilde{a}_{l \neq j} ; \frac{1}{\bar{z}}\right)\right),
\end{aligned}
$$

where

$$
c_{00}^{p}=\prod_{i=1}^{p} B\left(b_{i}-a_{i}, a_{i}-a_{0}\right)
$$

and

$$
c_{0 j}^{p}=B\left(a_{j}, a_{0}-a_{j}\right) \prod_{\substack{i=1 \\ i \neq j}}^{p} B\left(b_{i}-a_{i}, a_{i}-a_{j}\right),
$$

with $B(a, b)=\frac{\Gamma(a) \Gamma(b)}{\Gamma(a+b)}$. $\tilde{c}_{0 k}^{p}$ is obtained from $c_{0 k}^{p}$ by replacing $a_{0}, a^{p}$ and $b^{p}$ by $\tilde{a}_{0}, \tilde{a}^{p}$ and $\tilde{b}^{p}$ respectively. Likewise we find

$$
\sum_{j=1}^{p} \lambda_{j}^{p+1} z^{1-b_{J}} \bar{z}^{1-\tilde{b}_{j}}{ }_{p+1} F_{p}\left(a_{l}-b_{j}+1,1+b_{i}-b_{j}, 2-b_{j}, z\right)
$$




$$
\begin{aligned}
\times{ }_{p+1} & F_{p}\left(\tilde{a}_{l}-\tilde{b}_{j}+1,1+\tilde{b}_{i}-\tilde{b}_{j}, 2-\tilde{b}_{j} ; \frac{1}{\bar{z}}\right) \\
= & \sum_{j=1}^{p}(-1)^{a_{j}-\tilde{a}_{j}} \frac{s\left(\tilde{b}_{j}-1\right) s\left(1-\tilde{a}_{0}\right)}{s\left(\tilde{b}_{j}-\tilde{a}_{0}\right)} \\
& \times \prod_{\substack{i=1 \\
i \neq j}}^{p} \frac{s\left(\tilde{a}_{i}-\tilde{b}_{j}+1\right) s\left(\tilde{b}_{i}-\tilde{a}_{j}\right)}{s\left(\tilde{b}_{i}-\tilde{b}_{j}+1\right)} \\
& \times\left(\sum_{k=0}^{p} c_{j k}^{p}\left(\frac{e^{i \pi}}{z}\right)^{a_{k}}{ }_{p+1} F_{p}\left(a_{k}, 1+a_{k}-b_{i}, 1+a_{k}-a_{l \neq k} ; \frac{1}{z}\right)\right) \\
& \times\left(\left(\sum_{k=0}^{p} \tilde{c}_{j k}^{p}\left(\frac{e^{-i \pi}}{\bar{z}}\right)^{\tilde{a}_{k}}{ }_{p+1} F_{p}\left(\tilde{a}_{k}, 1+\tilde{a}_{k}-\tilde{b}_{i} 1+\tilde{a}_{k}-\tilde{a}_{l \neq k} ; \frac{1}{\bar{z}}\right)\right),\right.
\end{aligned}
$$

with

$$
c_{j 0}^{p}=\frac{\Gamma\left(2-b_{j}\right) \Gamma\left(b_{j}-1\right)}{\Gamma\left(b_{j}-a_{j}\right) \Gamma\left(a_{j}-b_{j}+1\right)} \prod_{i=1}^{p} B\left(b_{i}-a_{i}, a_{i}-a_{0}\right),
$$

and

$$
c_{j k}^{p}=\frac{\Gamma\left(2-b_{j}\right) \Gamma\left(b_{j}-1\right)}{\Gamma\left(b_{j}-a_{0}\right) \Gamma\left(a_{0}-b_{j}+1\right)} B\left(1-a_{0}, a_{0}-a_{k}\right) \prod_{\substack{i=1 \\ i \neq k}}^{p} B\left(b_{i}-a_{i}, a_{i}-a_{k}\right) \frac{s\left(b_{j}-a_{j}\right)}{s\left(b_{j}-a_{k}\right)} .
$$

With the simplifications

$$
\begin{gathered}
\frac{s\left(\tilde{a}_{i}-\tilde{b}_{j}+1\right)}{s\left(\tilde{b}_{i}-\tilde{b}_{j}+1\right)}=\frac{s\left(\tilde{a}_{i}-\tilde{b}_{j}\right)}{s\left(\tilde{b}_{i}-\tilde{b}_{j}\right)}, \\
\frac{s\left(\tilde{b}_{j}-1\right) \Gamma\left(2-\tilde{b}_{j}\right) \Gamma\left(\tilde{b}_{j}-1\right)}{s\left(\tilde{b}_{j}-\tilde{a}_{0}\right) \Gamma\left(\tilde{b}_{j}-\tilde{a}_{0}\right) \Gamma\left(\tilde{a}_{0}-\tilde{b}_{j}+1\right)}=1, \\
\frac{s\left(1-\tilde{a}_{0}\right)}{s\left(b_{j}-1\right)}=-\frac{s\left(\tilde{a}_{0}\right)}{s\left(b_{j}\right)},
\end{gathered}
$$

and

$$
B\left(1-a_{0}, a_{0}-a_{l}\right)=B\left(a_{l}, a_{0}-a_{l}\right) \frac{s\left(a_{l}\right)}{s\left(a_{0}\right)}
$$


the coefficient $J_{n k}, n \neq 0 \neq k$ of

$$
\begin{aligned}
& \left(\frac{e^{i \pi}}{z}\right)^{a_{n}}\left(\frac{e^{-i \pi}}{\bar{z}}\right)^{\tilde{a}_{k}}{ }_{p+1} F_{p}\left(a_{n}, a_{n}-b_{i}+1,1+a_{n}-a_{l \neq n} \text { frac } 1 z\right) \\
& \times_{p+1} F_{p}\left(\tilde{a}_{k}, \tilde{a}_{k}-\tilde{b}_{i}+1,1+\tilde{a}_{k}-\tilde{a}_{l \neq k} ; \frac{1}{\bar{z}}\right)
\end{aligned}
$$

becomes,

$$
\begin{aligned}
J_{n k}= & \prod_{\substack{i=0 \\
i \neq n}}^{p} B\left(b_{i}-a_{i}, a_{i}-a_{n}\right) \prod_{\substack{i=0 \\
i \neq k}}^{p} B\left(\tilde{b}_{i}-\tilde{a}_{i}, \tilde{a}_{i}-\tilde{a}_{k}\right) \\
& \times \prod_{i=1}^{p} \sin \pi\left(\tilde{b}_{i}-\tilde{a}_{i}\right) B\left(a_{n}, a_{0}-a_{n}\right) B\left(\tilde{a}_{k}, \tilde{a}_{0}-\tilde{a}_{k}\right) \hat{J}_{n k},
\end{aligned}
$$

where

$$
\begin{aligned}
\tilde{J}_{n k}= & \prod_{i=1}^{p} \frac{s\left(\tilde{a}_{i}\right)}{s\left(\tilde{b}_{i}\right)}-\sum_{j=1}^{p}(-1)^{a_{j}-\tilde{a}_{j}} \frac{s\left(b_{j}-a_{0}\right) s\left(a_{n}\right) s\left(\tilde{a}_{k}\right) s\left(b_{j}-a_{j}\right)}{s\left(b_{j}\right) s\left(a_{0}\right) s\left(b_{j}-a_{n}\right) s\left(\tilde{b}_{j}-\tilde{a}_{k}\right)} \\
& \times \prod_{\substack{p=1 \\
i \neq j}}^{p} \frac{s\left(\tilde{a}_{i}-\tilde{b}_{j}\right)}{s\left(\tilde{b}_{i}-\tilde{b}_{j}\right)} .
\end{aligned}
$$

Now from the fact that $a_{i}-\tilde{a}_{i}$ and $b_{i}-\tilde{b}_{i}$ differ by integers we find,

$$
\begin{aligned}
(-1)^{a_{j}-\tilde{a}_{j}} \frac{s\left(b_{j}-a_{0}\right) s\left(a_{n}\right) s\left(b_{j}-a_{j}\right)}{s\left(b_{j}\right) s\left(a_{0}\right) s\left(b_{j}-a_{n}\right)} & \\
=- & \frac{s\left(\tilde{b}_{j}-\tilde{a}_{0}\right) s\left(\tilde{a}_{0}\right) s\left(\tilde{a}_{n}\right) s\left(\tilde{a}_{j}-\tilde{b}_{j}\right)}{s\left(\tilde{b}_{j}\right) s\left(\tilde{a}_{0}\right) s\left(\tilde{b}_{j}-\tilde{a}_{n}\right)}
\end{aligned}
$$

so that

$$
\begin{aligned}
\tilde{J}_{n k}= & \prod_{i=1}^{p} \frac{s\left(\tilde{a}_{i}\right)}{s\left(\tilde{b}_{j}\right)} \\
& +\sum_{j=1}^{p} \frac{s\left(\tilde{b}_{j}-\tilde{a}_{0}\right) s\left(\tilde{a}_{n}\right) s\left(\tilde{a}_{k}\right) s\left(\tilde{a}_{j}-\tilde{b}_{j}\right)}{s\left(\tilde{b}_{j}\right) s\left(\tilde{a}_{0}\right) s\left(\tilde{a}_{n}-\tilde{b}_{j}\right) s\left(\tilde{a}_{k}-\tilde{b}_{j}\right)} \prod_{\substack{i=1 \\
i \neq j}}^{p} \frac{s\left(\tilde{a}_{i}-\tilde{b}_{j}\right)}{s\left(\tilde{b}_{i}-\tilde{b}_{j}\right)} .
\end{aligned}
$$


For $n=0 \neq k$ the coefficient $J_{0 k}$ of

$$
\begin{aligned}
& \left(\frac{e^{i \pi}}{z}\right)^{a_{0}}\left(\frac{e^{-i \pi}}{\bar{z}}\right)^{\tilde{a}_{k}}{ }_{p+1} F_{p}\left(a_{0}, 1+a_{0}-b_{i}, 1+a_{0}-a_{i}, \frac{1}{z}\right) \\
& \times{ }_{p+1} F_{p}\left(\tilde{a}_{k}, 1+\tilde{a}_{k}-\tilde{b}_{i}, 1+\tilde{a}_{k}-\tilde{a}_{l \neq k}, \frac{1}{z}\right)
\end{aligned}
$$

becomes, with the substitutions above,

$$
J_{0, k}=\prod_{i=1}^{p} B\left(b_{i}-a_{i}, a_{i}-a_{0}\right) \prod_{\substack{i=1 \\ i \neq k}}^{p} B\left(\tilde{b}_{i}-\tilde{a}_{i}, \tilde{a}_{i}-\tilde{a}_{k}\right) B\left(\tilde{a}_{k}, \tilde{a}_{0}-\tilde{a}_{k}\right) \hat{J}_{0, k}
$$

where $\tilde{J}_{0, k}$ is given by (18) above with $n=k$. For $k=0$ we find

$$
\begin{aligned}
J_{0,0}= & \prod_{i=1}^{p} B\left(b_{i}-a_{i}, a_{i}-a_{0}\right) \prod_{i=1}^{p} B\left(\tilde{b}_{i}-\tilde{a}_{i}, \tilde{a}_{i}-\tilde{a}_{i}\right) \prod_{i=1}^{p} s\left(\tilde{b}_{i}-\tilde{a}_{i}\right) \\
& \times\left[\prod_{i=1}^{p} \frac{s\left(\tilde{a}_{i}\right)}{s\left(\tilde{b}_{i}\right)}-\sum_{j=1}^{p} \frac{s\left(\tilde{a}_{0}\right) s\left(\tilde{b}_{j}-\tilde{a}_{j}\right)}{s\left(\tilde{b}_{j}\right) s\left(\tilde{b}_{j}-\tilde{a}_{j}\right)} \prod_{\substack{i=1 \\
i \neq j}}^{p} \frac{s\left(\tilde{a}_{i}-\tilde{b}_{j}\right)}{s\left(\tilde{b}_{i}-\tilde{b}_{j}\right)}\right] .
\end{aligned}
$$

With the substitutions $y_{j}=\exp -2 i \pi b_{j}, u_{i}=\exp -2 i \pi a_{i}$ and $B_{n, k}=J_{n, k} \prod_{i}^{p} \frac{\exp i \pi a_{i}}{\exp i \pi b_{i}}$ the result now follows from Lemma 3 and the symmetry between $J_{n, 0}$ and $J_{0, n}$.

Define

$$
\psi_{p}=\sum_{i=1}^{p} b_{i}-\sum_{i=0}^{p} a_{i}
$$

and $\tilde{\psi}_{p}$ as above with $b_{i}$ and $a_{i}$ replaced by $\tilde{b}_{i}$ and $\tilde{a}_{i}$ respectively.

Lemma 6 Suppose Condition $C$ is satisfied, $\psi_{p-1}$ is not an integer, $\operatorname{Re}\left(\psi_{p-1}+\tilde{\psi}_{p-1}\right)>$ $p-1, \operatorname{Re}\left(a_{p}+\tilde{a}_{p}\right)<0, \operatorname{Re}\left(b_{p}+\tilde{b}_{p}-a_{p}+\tilde{a}_{p}\right)<0, \operatorname{Re}\left(b_{j}+\tilde{b}_{j}-a_{p}-\tilde{a}_{p}\right)<2$, and $\operatorname{Re}\left(b_{p}+\tilde{b}_{p}-a_{j}-\tilde{a}_{j}\right)<2$. Then for $z \neq 0$ or 1

$$
\begin{aligned}
& \int_{\Gamma} d \bar{z}_{p} a_{0} \prod_{i=0}^{p-1} a_{i} K_{0}^{p}\left(a_{0}+1, a^{p}+1, b^{p}, z, z_{p}\right) H_{0}^{p}\left(\tilde{a}_{0}, \tilde{a}^{p}, \bar{z}, \bar{z}_{p}\right) \\
& +\prod_{i=0}^{p-1}\left(a_{i}-b_{j}+1\right) K_{j}^{p}\left(a_{0}+1, a^{p}+1, b^{p}, z, z_{p}\right) H_{j}^{p}\left(\tilde{a}_{0}, \tilde{a}^{p}, \tilde{b}^{p}, \bar{z}, \bar{z}_{p}\right)=0
\end{aligned}
$$

where $\Gamma=[0, \infty \exp -i \phi) \cup[1, \infty \exp -i \phi), \phi=\arg (z)$. 
Proof. We use $\Gamma$ a cuts for $z^{\alpha}$ and $(1-z)^{\alpha}$ choosing the determination for both so that both give positive reals when their arguments a large positive real numbers. We beginning by breaking up the above integral into pieces for which $\left|z z_{p}\right|<1$ and $\left|z z_{p}\right|>1$. For $\left|z z_{p}\right|>1$ we use the fact that

$$
\begin{aligned}
& \prod_{i=0}^{p-1} a_{i} \lambda_{0}^{p-1}\left(a_{0}, a^{p-1}, b^{p-1}, \tilde{a}_{0}, \tilde{a}^{p-1}, \tilde{b}^{p-1}\right) \\
& =\prod_{i=1}^{p-1}\left(b_{i}-a_{i}-1\right) \lambda_{0}^{p-1}\left(a_{0}+1, a^{p-1}+1, b^{p-1}, \tilde{a}_{0}, \tilde{a}^{p-1}, \tilde{b}^{p-1}\right),
\end{aligned}
$$

and

$$
\begin{aligned}
& \prod_{i=0}^{p-1}\left(a_{i}-b_{j}+1\right) \lambda_{j}^{p-1}\left(a_{0}, a^{p-1}, b^{p-1}, \tilde{a}_{0}, \tilde{a}^{p-1}, \tilde{b}^{p-1}\right) \\
& =a_{0} \prod_{i=1}^{p-1}\left(b_{i}-a_{i}-1\right) \lambda_{j}^{p-1}\left(a_{0}+1, a^{p-1}+1, b^{p-1}, \tilde{a}_{0}+1, \tilde{a}^{p-1}+1, \tilde{b}^{p-1}\right),
\end{aligned}
$$

and Lemma 5 to represent the integrand in $(22)$ for $\left|z z_{p}\right|>1$. The conditions on $a_{0} \ldots, a_{p}, b_{1} \ldots, a_{p}, \tilde{a}_{0} \ldots, \tilde{a}_{p}$, and $\tilde{b}_{1} \ldots, \tilde{b}_{p}$ imply that the above integral is convergent for $z_{p} \sim \infty, 0$, and 1. Furthermore with condition $C$ and the condition on $\psi_{p-1}$ the hypergeometric functions in the integrand behave as ${ }_{p} F_{p-1}\left(a_{0}, a^{p}, b^{p}, z\right) \sim(1-z)^{\psi_{p-1}}$ ([8]) so that the integral is convergent for $z z_{p} \sim 1$. The last two parts of condition $C$ insure that the contributions along the two sides of each cut cancel out giving the result.

We now prove our main result,

Theorem 3 Suppose that condition $C$ is satisfied, $\psi_{p-1}$ is not an integer, $\operatorname{Re}\left(\psi_{p-1}+\right.$ $\left.\tilde{\psi}_{p-1}\right)>-2, \operatorname{Re}\left(a_{p}+\tilde{a}_{p}\right)>0, \operatorname{Re}\left(b_{p}+\tilde{b}_{p}-a_{p}-\tilde{a}_{p}\right)>0, \operatorname{Re}\left(b_{j}+\tilde{b}_{j}-a_{p}-\tilde{a}_{p}\right)<2$, and $\operatorname{Re}\left(b_{p}+\tilde{b}_{p}-a_{j}-\tilde{a}_{j}\right)<2$. then $I_{p+1}$ is given by equation (8) with $\lambda_{j}^{p+1}$ given by (9) and (10). $I_{p+1}$ may be extended using the above representation so that only condition $C$ is satisfied by the parameters.

Proof. Consider $I_{p+1}$ for $p=1$, the result is already known ([4]) and we will recover it. In this case,

$$
I_{2}=
$$




$$
\begin{aligned}
& \iint_{\mathbb{C} \backslash \Gamma} \frac{d^{2} z_{1}}{2 i} z_{1}^{a_{1}-1}\left(1-z_{1}\right)^{b_{1}-a_{1}-1}\left(1-z z_{1}\right)^{-a_{0}} \bar{z}_{1}^{\tilde{a}_{1}-1}\left(1-\bar{z}_{1}\right)^{\tilde{b}_{1}-\tilde{a}_{1}-1}\left(1-\bar{z} \bar{z}_{1}\right)^{-\tilde{a}_{0}} \\
& =\iint_{\mathbb{C} \backslash \Gamma} \frac{d^{2} z_{1}}{2 i} z_{1}^{a_{1}-1}\left(1-z_{1}\right)^{b_{1}-a_{1}-1} \bar{z}_{1}^{\tilde{a}_{1}-1}\left(1-\bar{z}_{1}\right)^{\tilde{b}_{1}-\tilde{a}_{1}-1} I_{1}\left(a_{0}, \tilde{a}_{0}, z z_{1}, \bar{z} \bar{z}_{1}\right) \\
& =\iint_{\mathbb{C}} \frac{d^{2} z_{1}}{2 i} \chi_{\mathbb{C} \backslash \Gamma} z_{1}^{a_{1}-1}\left(1-z_{1}\right)^{b_{1}-a_{1}-1} \bar{z}_{1}^{\tilde{a}_{1}-1}\left(1-\bar{z}_{1}\right)^{\tilde{b}_{1}-\tilde{a}_{1}-1} \\
& \times I_{1}\left(a_{0}, \tilde{a}_{0}, z z_{1}, \bar{z} \bar{z}_{1}\right) .
\end{aligned}
$$

where $\Gamma=[0, \infty \exp -i \phi) \cup[1, \infty \exp -i \phi), \phi=\arg (z)$ and $\chi_{\mathbb{C} \backslash \Gamma}$ is the characteristic function of the set $\mathbb{C} \backslash \Gamma$. We will use the determination given in the above lemma for $z^{\alpha}$. We suppose that $z \neq 0$ or 1 . If $\operatorname{Re}\left(a_{1}+\tilde{a}_{1}\right)>0, \operatorname{Re}\left(b_{1}+\tilde{b}_{1}-a_{1}-\tilde{a}_{1}\right)>0$, $\operatorname{Re}\left(a_{0}+\tilde{a}_{0}\right)<2$, and $\operatorname{Re}\left(b_{1}+\tilde{b}_{1}-a_{0}-\tilde{a}_{0}\right)<2$ the above integral converges uniformly on compact subsets of the $z$ plane that exclude zero and one and defines an analytic function of the $a$ and $b$ variables. Utilizing the third line in the above equation it is not difficult to see that the integral is continuous in $z$. For $\epsilon$ small but positive let $I_{2}^{\epsilon}$ be given by the integral above with $\Gamma$ replaced by $\Gamma_{\epsilon}$ which is the contour with every point in $\Gamma$ displaced by a distance $\epsilon$. Thus

$$
I_{2}^{\epsilon}=\iint_{\mathbb{C}} d^{2} z_{1} \chi_{\mathbb{C} \backslash \Gamma_{\epsilon}} z_{1}^{a_{1}-1}\left(1-z_{1}\right)^{b_{1}-a_{1}-1} \bar{z}_{1}^{\tilde{a}_{1}-1}\left(1-\bar{z}_{1}\right)^{\tilde{b}_{1}-\tilde{a}_{1}-1} I_{1}\left(a_{0}, \tilde{a}_{0}, z z_{1}, \bar{z} \bar{z}_{1}\right) .
$$

The integral converges uniformly for $z$ on compact subsets contained in or on $\Gamma_{\epsilon}$ that avoid $z=0,1$ and defines an analytic function in the variables $a_{0}, a_{1}, b_{1}, \tilde{a}_{0}, \tilde{a}_{1}, \tilde{b}_{1}$ in the region designated above. Furthermore it follows that $I_{2}^{\epsilon}$ is a continuous function of $\epsilon$ for $z$ sufficiently close to $\Gamma \backslash\{0,1\}$ and $\lim _{\epsilon \rightarrow 0} I_{2}^{\epsilon}=I_{2}$ where the convergence is uniform on compact subsets of $\Gamma \backslash\{0,1\}$. Since for every $\epsilon$ small but positive the integral, and the integral with the integrand differentiated once, and the integral with the integrand differentiate twice with respect to $z$ converge uniformly, it is possible to interchange integration and differentiation with respect to $z$. From (12) above we find

$$
\begin{aligned}
O_{z}^{2} I_{2}^{\epsilon}(z, \bar{z})= & -a_{0} \frac{2}{2 i} \int d^{2} z_{1} \frac{\partial}{\partial z_{1}} K_{0}^{(1)}\left(a_{0}+1, a_{1}+1, b_{1}, z, z_{1}\right) \\
& H_{0}^{(1)}\left(\widetilde{a}_{0}, \widetilde{a}_{1}, \tilde{b}_{1}, \bar{z}, \bar{z}_{1}\right)
\end{aligned}
$$

Thus from Stokes' Theorem we find

$$
O_{z}^{2} I_{2}^{\epsilon}=\frac{1}{4} \int_{\Gamma_{\epsilon}} K_{0}^{(1)}\left(z, z_{1}\right) H\left(\bar{z}, \bar{z}_{1}\right) d \bar{z}_{1}
$$




$$
=G_{\epsilon}(z, \bar{z})
$$

To continue on we impose the extra restriction that $\operatorname{Re}\left(a_{0}+\tilde{a}_{0}\right)<0$. Under these conditions it follows using Stokes' Theorem that for $z$ sufficiently close to $\Gamma \backslash\{0,1\}, G_{\epsilon}(z, \bar{z})$ is continuous in $\epsilon$. From Lemma 6 we also see that $\lim _{\epsilon \rightarrow 0} G_{\epsilon}(z, \bar{z})=0$ uniformly on compact subsets of $\Gamma \backslash\{0,1\}$. Thus we can conclude that for $z$ inside $\Gamma_{\epsilon}$.

$$
I_{\epsilon}^{2}(z)=\psi_{h}(z)+\psi_{p}(z)
$$

where $\psi_{h}(z)=c_{0}^{\epsilon} u_{0}^{2}+c_{1}^{\epsilon} u_{1}^{2}$ and

$$
\psi_{p}(z)=\sum_{i=0}^{1} u_{i}^{2}(z) \int_{z_{0}}^{z} \frac{w_{i}\left(u_{0}^{2}, u_{1}^{2}\right)(u)}{w\left(u_{0}^{2}, u_{1}^{2}\right)(u)} G_{\epsilon}(u) d u
$$

where $w\left(u_{0}^{2}, u_{1}^{2}\right)$ is the Wronskian of $u_{0}^{2}$ and $u_{1}^{2}$ while $w_{i}\left(u_{0}^{2}, u_{1}^{2}\right)$ is the determinant obtained from $w\left(u_{0}^{2}, u_{1}^{2}\right)$ by replacing the $i$ th column of $w$ by $\left(\begin{array}{l}0 \\ 1\end{array}\right)$. Condition $C$ insures that $u_{0}^{2}$ and $u_{1}^{2}$ are linearly independent. Here $z_{0}$ is chosen so that $0<\left|z_{0}\right|<1$ and $z_{0}$ lies on $\Gamma$. The coefficients $c_{0}^{\epsilon}$ and $c_{1}^{\epsilon}$ are functions of $\bar{z}$ (thought of as independent of $z$ for their computation). A similar discussion interchanging $z, a_{0}, a_{1}$, and $b_{1}$ with $\bar{z}, \tilde{a}_{0}, \tilde{a}_{1}$, and $\tilde{b}_{1}$ respectively shows that

$$
I_{2}^{\epsilon}=\sum_{i=0, j=0}^{1} \beta_{i, j}^{\epsilon} u_{i}^{2}\left(a_{0}, a_{1}, b_{1}, z\right) u_{j}^{2}\left(\tilde{a}_{0}, \tilde{a}_{1}, \tilde{b}_{1}, \bar{z}\right)+f^{\epsilon},
$$

where $f^{\epsilon}$ is a function of $u_{0,1}^{2}\left(a_{0}, a_{1}, b_{1}, z\right), u_{0,1}^{2}\left(\tilde{a}_{0}, \tilde{a}_{1}, \tilde{b}_{1}, \bar{z}\right)$ and their first derivatives. The theory of differential equations [9 Theorem 4.1] says that the $\beta_{i, j}^{\epsilon}$ and $f^{\epsilon}$ are continuous functions of $\epsilon$ for $z \in \Gamma \backslash\{0,1\}$ and $\lim _{\epsilon \rightarrow 0} f^{\epsilon}=0$ uniformly on compact subsets of $\Gamma \backslash\{0,1\}$. Writing

$$
\begin{aligned}
& I_{2}= \\
& \iint_{\substack{\mathbb{C} \backslash \Gamma \\
\left|z_{1}\right| \leq 1}} \frac{d^{2} z_{1}}{2 i} z_{1}^{a_{1}-1}\left(1-z_{1}\right)^{b_{1}-a_{1}-1}\left(1-z z_{1}\right)^{-a_{0}} \bar{z}_{1}^{\tilde{a}_{1}-1}\left(1-\bar{z}_{1}\right)^{\tilde{b}_{1}-\tilde{a}_{1}-1}\left(1-\bar{z} \bar{z}_{1}\right)^{-\tilde{a}_{0}} \\
& +\iint_{\substack{\mathbb{C} \Gamma \\
\left|z_{1}\right|>1}} \frac{d^{2} z_{1}}{2 i} z_{1}^{a_{1}-1}\left(1-z_{1}\right)^{b_{1}-a_{1}-1}\left(1-z z_{1}\right)^{-a_{0}} \bar{z}_{1}^{\tilde{a}_{1}-1}\left(1-\bar{z}_{1}\right)^{\tilde{b}_{1}-\tilde{a}_{1}-1}\left(1-\bar{z} \bar{z}_{1}\right)^{-\tilde{a}_{0}} \\
& =I_{1}^{2}+I_{2}^{2} \text {. }
\end{aligned}
$$


With the above constraints on the parameters we see that in a neighborhood of $z=0$, $I_{2}^{1} \sim K$ where $K$ is a constant independent of $z$ and $\bar{z}$. The change of variables $t=z z_{1}$ in the second integral yields

$$
I_{2}^{2}=z^{1-b_{1}} \bar{z}^{1-\tilde{b}_{1}}(-1)^{b_{1}-\tilde{b}_{1}-a_{1}+\tilde{a}_{1}} \int_{\substack{\mathbb{C} \backslash[0 \infty) \\\left|\frac{t}{z}\right|>1}} \int_{\frac{d}{2} t} \frac{d^{b_{1}-2}}{2 i} t^{b_{1}}\left(1-\frac{z}{t}\right)^{b_{1}-a_{1}-1}(1-t)^{-a_{0}} \bar{t}^{\tilde{b}_{1}-2}\left(1-\frac{\bar{z}}{\bar{t}}\right)^{\tilde{b}_{1}-\tilde{a}_{1}-1}(1-\bar{t})^{-\tilde{a}_{0}} .
$$

Consequently the dominated convergence theorem shows that

$$
\lim _{z \rightarrow 0} z^{b_{1}-1} \bar{z}^{\tilde{b}_{1}-1} I_{2}^{2}=\lambda_{1}^{2}
$$

Since the only constraints on $b_{1}$ and $\tilde{b}_{1}$ are those give above and condition $C$ the above considerations imply that $\beta_{0,1}^{0}=0=\beta_{1,0}^{0}$ and $\beta^{1,1}=\lambda_{1}^{2}$. We also find from the dominated convergence theorem that for $z$ real $\lim _{z \rightarrow 0} I_{2}=\lambda_{0}^{2}$. From the previous discussion the solution reads

$$
\begin{aligned}
& I_{2}^{\epsilon}\left(a_{0}, a_{1}, b_{1}, \tilde{a}_{0}, \tilde{a}_{1}, \tilde{b}_{1}, z, \bar{z}\right) \\
= & \lambda_{0}^{(2)}\left(a_{1}, b_{1} ; \tilde{a}_{1}, \tilde{b}_{1}\right){ }_{2} F_{1}\left(a_{0}, a_{1}, b_{1} ; z\right){ }_{2} F_{1}\left(\tilde{a}_{0}, \tilde{a}_{1}, \tilde{b}_{1} ; \bar{z}\right) \\
& +\lambda_{1}^{(2)}\left(a_{0}, a_{1}, b_{1}, \tilde{a}_{0}, \tilde{a}_{1}, \tilde{b}_{1}\right)(z)^{1-b_{1}}(\bar{z})^{1-\tilde{b}_{1}} \\
& { }_{2} F_{1}\left(a_{0}-b_{1}+1, a_{1}-b_{1}+1,2-b_{1} ; z\right) \\
& { }_{2} F_{1}\left(\tilde{a}_{0}-\tilde{b}_{1}+1, \tilde{a}_{1}-\tilde{b}_{1}+1,2-\tilde{b}_{1} ; \bar{z}\right) \\
& \lambda_{0}^{(2)}\left(a_{1}, b_{1}, \tilde{a}_{1}, \tilde{b}_{1}\right) \\
= & \frac{\Gamma\left(a_{1}\right) \Gamma\left(b_{1}-a_{1}\right)}{\Gamma\left(b_{1}\right)} \frac{\Gamma\left(\tilde{a}_{1}\right) \Gamma\left(\tilde{b}_{1}-\tilde{a}_{1}\right)}{\Gamma\left(\tilde{b}_{1}\right)} \frac{\sin \pi \tilde{a}_{1} \sin \pi\left(\tilde{b}_{1}-\tilde{a}_{1}\right)}{\sin \pi \tilde{b}_{1}}
\end{aligned}
$$

and

$$
\begin{aligned}
& \lambda_{1}^{(2)}\left(a_{0}, a_{1}, b_{1}, \tilde{a}_{0}, \tilde{a}_{1}, \tilde{b}_{1}\right) \\
= & (-1)^{b_{1}-\tilde{b}_{1}+a_{1}-\tilde{a}_{1}} \frac{\Gamma\left(b_{1}-1\right) \Gamma\left(-a_{0}+1\right)}{\Gamma\left(-a_{0}+b_{1}\right)} \frac{\Gamma\left(\tilde{b}_{1}-1\right) \Gamma\left(-\tilde{a}_{0}+1\right)}{\Gamma\left(-\tilde{a}_{0}+\tilde{b}_{1}\right)} \\
& \times \frac{\sin \pi b_{1} \sin \pi\left(-a_{0}\right)}{\sin \pi\left(b_{1}-a_{0}\right)}
\end{aligned}
$$


in agreement with previous results. This proves the result for $p=1$ for $\operatorname{Re}\left(a_{1}+\tilde{a}_{1}\right)>0$, $\operatorname{Re}\left(b_{1}+\tilde{b}_{1}-a_{1}-\tilde{a}_{1}\right)>0, \operatorname{Re}\left(a_{0}+\tilde{a}_{0}\right)<0$, and $\operatorname{Re}\left(b_{1}+\tilde{b}_{1}-a_{0}-\tilde{a}_{0}\right)<2$. We may now extend $I_{2}$ using the above representation so that only condition $C$ is satisfied by the parameters.

Suppose now that,

$$
\begin{aligned}
& I_{p}\left(a_{0}, a^{p-1}, b^{p-1}, \tilde{a}_{0}, \tilde{a}^{p-1}, \tilde{b}^{p-1}, z, \bar{z}\right) \\
= & \sum_{j=0}^{p-1} \lambda_{j}^{(p)}\left(a_{0}, a^{p-1}, b^{p-1}, \tilde{a}_{0}, \tilde{a}^{p-1}, \tilde{b}^{p-1}\right) u_{j}^{p}\left(a_{0}, a^{p-1}, b^{p-1}, z\right) \times \\
& \times \tilde{u}_{j}^{p}\left(\tilde{a}_{0}, \tilde{a}^{p-1} \tilde{b}^{p-1}, \bar{z}\right),
\end{aligned}
$$

then

$$
\begin{aligned}
& I_{p+1}\left(a_{0}, a^{p}, b^{p}, \tilde{a}_{0}, \tilde{a}^{p}, \tilde{b}^{p}, z, \bar{z}\right) \\
= & \sum_{j=0}^{p-1} \lambda_{j}^{(p)}\left(\tilde{a}_{0}, \tilde{a}^{p-1}, \tilde{b}^{p-1}, \tilde{a}_{0}, \tilde{a}^{p-1}, \tilde{b}^{p-1}\right) \times \\
& \times \frac{1}{2 i} \iint_{\mathbb{C} \backslash \Gamma} d^{2} z_{1}\left(z_{1}\right)^{a_{p}-1}\left(1-z_{1}\right)^{b_{p}-a_{p}-1} u_{j}^{p}\left(a_{0}, a_{i}, b_{i}, z z_{1}\right) \\
& \left(\bar{z}_{1}\right)^{\tilde{a}_{p}-1}\left(1-\bar{z}_{1}\right)^{\tilde{b}_{p}-\tilde{a}_{p}-1} u_{j}^{p}\left(\tilde{a}_{0}, \tilde{a}^{p-1}, \tilde{b}^{p-1}, \bar{z} \bar{z}_{1}\right) .
\end{aligned}
$$

The conditions imposed above imply that $I_{p+1}$ is analytic in the parameters $a_{0}, a^{p}, b^{p}$, $\tilde{a}_{0}, \tilde{a}^{p}$, and $\tilde{b}^{p}$ in the region specified. Furthermore $I_{p+1}$ is a continuous function of $z$ for $z \neq 0,1$. We now impose the condition that $\operatorname{Re}\left(\psi_{p-1}+\tilde{\psi}_{p-1}\right)>p-1$. Let $\Gamma^{\epsilon}$ be as above and set

$$
\begin{aligned}
& I_{p+1}^{\epsilon}= \\
& \iint_{\mathbb{C}} d^{2} z_{1} \chi_{\mathbb{C} \backslash \Gamma_{\epsilon}} z_{1}^{a_{p}-1}\left(1-z_{1}\right)^{b_{p}-a_{p}-1} \bar{z}_{1} \tilde{a}_{p}-1\left(1-\bar{z}_{1}\right)^{\tilde{b}_{p}-\tilde{a}_{p}-1} \\
& \times I_{p}\left(a_{0}, a^{p-1}, b^{p-1} \tilde{a}_{0}, \tilde{a}^{p-1}, \tilde{b}^{p-1} z z_{1}, \bar{z} \bar{z}_{1}\right),
\end{aligned}
$$

and a discussion similar to that given above shows that $\lim _{\epsilon \rightarrow 0} I_{p+1}^{\epsilon}=I_{p+1}$ uniformly on compact subsets of $\Gamma \backslash\{0,1\}$. Since $I_{p+1}^{\epsilon}$ can be differentiated twice we find using Stokes' Theorem that 


$$
\begin{aligned}
& O_{z}^{p+1} I_{p+1}\left(a_{i}, b_{i}, \tilde{a}_{i}, b_{i}, a_{0}, \tilde{a}_{0}, z, \bar{z}\right) \\
= & -a_{0} \prod_{i=1}^{p-1}\left(b_{i}-a_{i}-1\right) \frac{1}{2 i} \int_{\mathbb{C} \backslash \Gamma_{\epsilon}} d z_{1} \frac{\partial}{\partial z_{1}} z_{1}^{a_{p}}\left(1-z_{1}\right)^{b_{p}-a_{p}} \\
& I_{p}\left(a_{0}+1, a_{i}+1, b_{i}, \tilde{a}_{0}, \tilde{a}_{i}, \tilde{b}_{i}, z z_{1}, z \bar{z}_{1}\right) d \bar{z}_{1} \bar{z}_{p}^{a_{p}-1}\left(1-\bar{z}_{1}\right)^{\tilde{b}_{p}-\tilde{a}_{p}-1} \\
= & -\frac{a_{0} \prod_{i=1}^{p-1}\left(b_{i}-a_{i}-1\right)}{4} d \bar{z}_{\epsilon} K_{0}^{p}\left(a_{0}+1, a^{p}+1, b^{p}, z, z_{1}\right) H_{0}^{p}\left(\tilde{a}_{0}, \tilde{a}^{p}, \bar{z}, \bar{z}_{1}\right) \\
& +K_{j}^{p}\left(a_{0}+1, a^{p}+1, b^{p}, z, z_{1}\right) H_{j}^{p}\left(\tilde{a}_{0}, \tilde{a}^{p}, \tilde{b}^{p}, \bar{z}, \bar{z}_{1}\right) \\
= & G_{p+1}^{\epsilon} .
\end{aligned}
$$

Lemma 5 shows that the last integral is convergent and $\lim _{\epsilon \rightarrow 0} G_{p+1}^{\epsilon}=0$ uniformly on compact subsets of $\Gamma \backslash\{0,1\}$ by Lemma 6 . A similar equation is obtained when using $O_{\bar{z}}^{p+1}$ and we are led to the solution

$$
I_{p+1}^{\epsilon}=\sum_{i, j=0}^{p+1} \beta_{i, j}^{\epsilon} u_{i}^{p+1}\left(a_{0}, a^{p}, b^{p}, z\right) u_{j}^{p+1}\left(\tilde{a}_{0}, \tilde{a}^{p}, \tilde{b}^{p} \bar{z}\right)+f^{\epsilon},
$$

where condition $C$ insures that the $u_{j}^{p+1}, j=0, \ldots, p$ are linearly independent. We again appeal to the theory of differential equations to show that $\lim _{\epsilon \rightarrow 0} f^{\epsilon}=0$ and that $\beta_{i, j}^{\epsilon}$ is a continuous function of $\epsilon$. Using the above continuity properties we find that $I_{p+1}=\sum_{i, j=0}^{p+1} \beta_{i, j} u_{i}^{p+1} u_{j}^{p+1}$. Write $I_{p+1}=\sum_{j=0}^{p} I_{p+1}^{j}$, where,

$$
\begin{aligned}
I_{p+1}^{j}=\frac{\lambda_{j}^{p}}{2 i} \iint_{\mathbb{C} \backslash \Gamma} & d^{2} z_{1} \chi\left|z z_{1}\right|<1 \\
& \times u_{j}^{p}\left(z_{1}\right) z_{1}^{a_{p}-1} a^{p-1}, \tilde{z}_{1}^{p-1}\left(1-z z_{1}\right)^{b_{p}-a_{p}-1}\left(1-\bar{z}_{1}\right)^{p}\left(\tilde{a}_{0}, \tilde{a}^{p-1}, \tilde{b}^{p-1}, \bar{z}_{p} \bar{z}_{1}\right),
\end{aligned}
$$

for $j=0, \ldots, p-1$, and

$$
\begin{aligned}
& I_{p+1}^{p}= \\
& \sum_{j=0}^{p-1} \frac{J_{j j} e^{i \pi\left(a_{j}-\tilde{a}_{j}\right)}}{2 i} \int_{\substack{|c| \Gamma \\
\mid z z_{1}>1}} d^{2} z_{1} z_{1}^{a_{p}-1} \bar{z}_{1}^{\tilde{a}_{p}-1}\left(1-z_{1}\right)^{b_{p}-a_{p}-1}\left(1-\bar{z}_{1}\right)^{\tilde{b}_{p}-\tilde{a}_{p}-1}\left(z z_{1}\right)^{-a_{j}}\left(\bar{z} \bar{z}_{1}\right)^{-\tilde{a}_{j}} \\
& \times{ }_{p} F_{p-1}\left(a_{j}, a_{j}-b_{i}+1,1+a_{j}-a_{i \neq j}, \frac{1}{z z_{1}}\right)_{p} F_{p-1}\left(\tilde{a}_{j}, \tilde{a}_{j}-\tilde{b}_{i}+1,1+\tilde{a}_{j}-\tilde{a}_{i \neq j}, \frac{1}{\bar{z} \bar{z}_{1}}\right) .
\end{aligned}
$$


From the dominated convergence theorem we see that $\lim _{z \rightarrow 0} I_{p+1}^{0}=\lambda_{0}^{p+1}$ and $\lim _{z \rightarrow 0} z^{b_{j}-1} \bar{z}^{\tilde{b}_{j}-1} I_{p+1}^{j}=\lambda_{j}^{p+1}$ for $z$ real. Furthermore with the change of variables $t=$ $z z_{1}$ it is not difficult to see that $\lim _{z \rightarrow 0} z^{b_{p}-1} \bar{z}^{\tilde{b}_{p}-1} I_{p+1}^{p}=K^{p+1}$, with $K^{p+1}$ independent of $z$. Condition $C$ and the above discussion shows that $\beta_{i, j}=0, i \neq j$ and $\beta_{i, i}=$ $\lambda_{i}^{p+1}, i=0, \ldots, p-1$. In order to compute $\beta_{p, p}$ split $I_{p+1}$ up into the pieces $I_{p+1}^{a}$ for $\left|z_{1}\right| \leq 1$ and $I_{p+1}^{b}$ for $\left|z_{1}\right|>1$. With the change of variables $t=z z_{1}$ the second integral becomes,

$$
\begin{aligned}
I_{p+1}^{b}=z^{1-b_{p}} \bar{z}^{1-\tilde{b}_{p}} \int_{\mathbb{C} \backslash[0, \infty)} \int d^{2} t \chi_{|t|>|z|}(t) t^{2-b_{p}} \bar{t}^{2-\tilde{b}_{p}}\left(\frac{z}{t}-1\right)^{b_{p}-a_{p}-1}\left(\frac{\bar{z}}{\bar{t}}-1\right)^{\tilde{b}_{p}-\tilde{a}_{p}-1} \\
\quad \times I_{p}\left(a_{0}, a^{p-1}, b^{p-1}, \tilde{a}_{0}, \tilde{a}^{p-1}, \tilde{b}^{p-1}, t, \bar{t}\right) .
\end{aligned}
$$

Using the dominated convergence theorem we see that,

$$
\begin{aligned}
& (-1)^{b_{p}-\tilde{b}_{p}-a_{p}+\tilde{a}_{p}} \lim _{z \rightarrow 0} z^{b_{p}-1} \bar{z}^{\tilde{b}_{p}-1} I_{p+1}^{b} \\
= & \int_{\mathbb{C} \backslash[0, \infty)} \int_{d^{2}} d^{b_{p}-2} \bar{t}^{\tilde{b}_{p}-2} I_{p}\left(a_{0}, a^{p-1}, b^{p-1}, \tilde{a}_{0}, \tilde{a}^{p-1}, \tilde{b}^{p-1}, t, \bar{t}\right) .
\end{aligned}
$$

We now show that the above integral is equal to $(-1)^{b_{p}-\tilde{b}_{p}-a_{p}+\tilde{a}_{p}} \lambda_{p}^{p+1}$. Note that the result has been shown for $p=1$. Now suppose the result is true up to $p$. With the substitution of (5) for $I_{p}$ we find,

$$
\begin{aligned}
& (-1)^{b_{p}-\tilde{b}_{p}-a_{p}+\tilde{a}_{p}} \lim _{z \rightarrow 0} z^{b_{p}-1} \bar{z}^{\tilde{b}_{p}-1} I_{p+1}^{b} \\
= & \int_{\mathbb{C} \backslash[0, \infty)} \int_{0} d^{2} t t^{b_{p}-2} \tilde{t}^{\tilde{b}_{p}-2} \iint_{\mathbb{C} \backslash \Gamma} d^{2} z z^{a_{p-1}-1} \bar{z}^{\tilde{a}_{p-1}-1}(1-z)^{b_{p-1}-a_{p-1}-1}(1-\bar{z})^{\tilde{b}_{p-1}-\tilde{a}_{p-1}-1} \\
& I_{p-1}\left(a_{0}, a^{p-2}, b^{p-2}, \tilde{a}_{0}, \tilde{a}^{p-2}, \tilde{b}^{p-2}, t z, \bar{t} z\right) .
\end{aligned}
$$

Decompose the second integral into the regions $R_{1}=|z| \leq 1$ and $R_{2}=|z|>1$ then make the change of variables $y=z t$. The integral $K_{1}$ over $R_{1}$ becomes,

$$
\begin{aligned}
K_{1}= & \int_{\mathbb{C} \backslash[0, \infty)} \int_{0} d^{2} t t^{b_{p}-2} \bar{t}^{\tilde{b}_{p}-2} \int_{\mathbb{C} \backslash[0, \infty)} \int_{1} d^{2}\left(\frac{y}{t}\right) \chi_{B}\left(\frac{y}{t}\right)\left(\frac{y}{t}\right)^{a_{p-1}-1}\left(\frac{\bar{y}}{\bar{t}}\right)^{\tilde{a}_{p-1}-1} \\
& \left(1-\frac{y}{t}\right)^{b_{p-1}-a_{p-1}-1}\left(1-\frac{\bar{y}}{\bar{t}}\right)^{\tilde{b}_{p-1}-\tilde{a}_{p-1}-1} I_{p-1}\left(a_{0}, a^{p-2}, b^{p-2}, \tilde{a}_{0}, \tilde{a}^{p-2}, \tilde{b}^{p-2}, y, \bar{y}\right),
\end{aligned}
$$


where $B$ the closed unit ball in complex plane. Interchanging the order of integration then setting $y=u t$ yields,

$$
\begin{aligned}
K_{1}= & \int_{\mathbb{C} \backslash[0, \infty)} \int_{2} d^{2} u \chi_{B}(u) u^{a_{p-1}-b_{p}} \bar{u}^{\tilde{a}_{p-1}-\tilde{b}_{p}}(1-u)^{b_{p-1}-a_{p-1}-1}(1-\bar{u})^{\tilde{b}_{p-1}-\tilde{a}_{p-1}-1} \\
& \iint_{\mathbb{C} \backslash \Gamma} d^{2} t(u t)^{b_{p}-2}(\bar{u} \bar{t})^{\tilde{b}_{p}-2} I_{p-1}\left(a_{0}, a^{p-2}, b^{p-2}, \tilde{a}_{0}, \tilde{a}^{p-2}, \tilde{b}^{p-2}, u t, \bar{u} \bar{t}\right),
\end{aligned}
$$

where $\Gamma$ is the cut described at the beginning of the Theorem with $\phi=\arg t$. Finally with the change of variable $w=u t$ we arrive at,

$$
\begin{aligned}
K_{1}= & \int_{\substack{\mathbb{C} \backslash[0, \infty) \\
|u| \leq 1}} d^{2} u u^{a_{p-1}-b_{p}-1} \bar{u}^{\tilde{a}_{p-1} \tilde{b}_{p}-1}(1-u)^{b_{p-1}-a_{p-1}-1}(1-\bar{u})^{\tilde{b}_{p-1}-\tilde{a}_{p-1}-1} \\
& \int_{\mathbb{C} \backslash \Gamma} \int_{\Gamma} d^{2} w w^{b_{p}-2} \bar{w}^{\tilde{b}_{p}-2} I_{p-1}\left(a_{0}, a^{p-2}, b^{p-2}, \tilde{a}_{0}, \tilde{a}^{p-2}, \tilde{b}^{p-2}, w, \bar{w}\right) .
\end{aligned}
$$

Applying the same operations to the integral over region $R_{2}$ and utilizing the fact

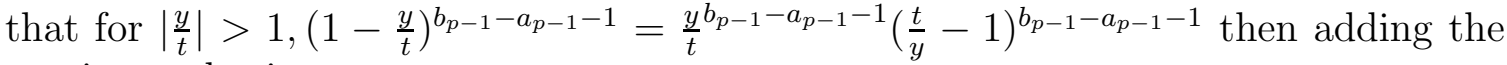
two integrals gives,

$$
\begin{aligned}
& (-1)^{b_{p}-\tilde{b}_{p}-a_{p}+\tilde{a}_{p}} \lim _{z \rightarrow 0} z^{b_{p}-1} \bar{z}^{\tilde{b}_{p}-1} I_{p+1}^{b} \\
= & \int_{\mathbb{C} \backslash \Gamma^{\prime}} d^{2} z z^{a_{p-1}-1} \bar{z}^{\tilde{a}_{p-1}-1}(1-z)^{b_{p-1}-a_{p-1}-1}(1-\bar{z})^{\tilde{b}_{p-1}-\tilde{a}_{p-1}-1} \\
& B_{b_{p}-1,-a_{0}+1 ; \tilde{b}_{p}-1,-\widetilde{a}_{0}+1} \prod_{i=1}^{p-2} B_{a_{i}-b_{p}+1, b_{i}-a_{i} ; \tilde{a}_{i}-\widetilde{b}_{p}+1, \widetilde{b}_{i}-\widetilde{a}_{i},}
\end{aligned}
$$

where the induction hypothesis has been. The claim now follows.

We may now extend the result to the case when $\operatorname{Re}\left(\psi_{p-1}+\tilde{\psi}_{p-1}\right)>-2$. The above representation may be used to define $I_{p+1}$ when only condition $C$ is imposed on the parameters which completes the proof.

A comment is in order. The result of this integral for $|z|<1$ is easier to get by using a new basis namely the ${ }_{p+1} G_{p}\left(a_{i} ; b_{i} ; z\right)$, which are nothing but the hypergeometric functions ${ }_{p+1} F_{p}\left(a_{i} ; b_{i} ; z\right)$ up to a multiplicative factor namely

$$
{ }_{p+1} G_{p}\left(a_{i} ; b_{i} ; z\right)=\frac{\prod_{0}^{p} \Gamma\left(a_{i}\right)}{\prod_{1}^{p} \Gamma\left(b_{i}\right)}{ }_{p+1} F_{p}\left(a_{i} ; b_{i} ; z\right)
$$


The basis of the expansion is now $V_{j}(z), \tilde{V}_{j}(\bar{z})$ rather than $U_{j}(z) \tilde{U}_{j}(\bar{z})$ where

$$
V_{j}(z)=z^{1-b_{j}}{ }_{p+1} G_{p}\left(a_{i}-b_{j}+1 ; b_{k}-b_{j}+1 ; z\right)
$$

and

$$
\tilde{V}_{j}(\bar{z})=z^{1-\tilde{b}_{j}}{ }_{p+1} \tilde{G}_{p}\left(\tilde{a}_{i}-\tilde{b}_{j}+1 ; \tilde{b}_{k}-\tilde{b}_{j}+1 ; \bar{z}\right)
$$

In these formulas $k \neq j=0, p$ where by convention $b_{0}=\tilde{b}_{0}=1$.

In this basis the solution reads

$$
\left.I=\sum_{j=0}^{p} \mu_{j} V_{j}(z) \tilde{V}_{(} \bar{z}\right)
$$

where the $\mu_{j}$ are obtained from the corresponding values of the $\lambda_{j}$.

It turns out that an obvious factorization does occur. Namely, by defining

$$
\mu_{p+1}=\frac{1}{\pi^{2}}(-1)^{a_{0}-\tilde{a}_{0}} \prod_{i=0}^{p} \Gamma\left(b_{i}-a_{i}\right) \Gamma\left(\tilde{b}_{i}-\tilde{a}_{i}\right) S\left(b_{i}-a_{i}\right)
$$

we get

$$
\mu_{j}=\mu_{p+1} \frac{\prod_{i=0}^{p} S\left(b_{j}-a_{i}\right)}{\prod_{i=0 \neq j}^{p} S\left(b_{j}-b_{i}\right)}
$$

To be more specific

$$
\begin{aligned}
& \mu_{0}=\mu_{p+1} \frac{\prod_{i=0}^{p} S\left(a_{i}\right)}{\prod_{i=1}^{p} S\left(b_{i}\right)} \\
& \mu_{j}=-\mu_{p+1} \frac{\prod_{i=0}^{p} S\left(b_{j}-a_{i}\right)}{S\left(b_{j}\right) \prod_{i=1 \neq j}^{p} S\left(b_{j}-b_{i}\right)}
\end{aligned}
$$

Similarly, for $|z|>1$ it is useful to expand the integral as a combination of hypergeometric functions which are defined for $|z|>1$ namely

$$
(z)^{-a_{j}}{ }_{p+1} G_{p}\left(a_{j}, a_{j}-b_{i}+1 ; a_{j}-a_{i}+1 ; \frac{1}{z}\right)
$$

or more specifically

$$
W_{j}(z)=(z)^{-a_{j}}{ }_{p+1} G_{p}\left(a_{j}, a_{j}-b_{i}+1 ; a_{j}-a_{i}+1 ; \frac{1}{z}\right)
$$


the solution reads now

$$
I=\sum_{j=1}^{p} \nu_{j} W_{j}(z) \tilde{W}_{j}(\bar{z})
$$

where the $\nu_{j}$ have to be determined from the value of the integral for $z \rightarrow \infty$.

We proceed as before and it turns out that the same factorisation does occur namely

$$
\nu_{j}=(-1)^{s-\tilde{s}} \mu_{p+1} \frac{\prod_{i=0}^{p} S\left(b_{i}-a_{i}\right)}{\prod_{i=1 \neq j}^{p} S\left(a_{i}-a_{j}\right)}
$$

Comparing $\mu_{j}$ and $\nu_{j}$ amounts but for a sign to interchange $b_{k}$ and $a_{k}$ as expected with the interchange of $z \rightarrow \frac{1}{z}$. Indeed if in the integrand we change $z_{i} \rightarrow \frac{1}{z_{i}}$ the new integrand $J$ reads

$$
\begin{aligned}
J= & (-1)^{s-\tilde{s}} z^{-a_{0}} \bar{z}^{-\tilde{a}_{0}} \prod_{i=1}^{p} t_{i}^{a_{0}-b_{i}}\left(1-t_{i}\right)^{b_{i}-a_{i}-1}\left(1-\prod_{i=1}^{p} t_{i} z^{-1}\right)^{-a_{0}} \\
& \times \prod_{i=1}^{p} \bar{t}_{i}^{\tilde{a}_{0}-\tilde{b}_{i}}\left(1-\bar{t}_{i}\right)^{\tilde{b}_{i}-\tilde{a}_{i}-1}\left(1-\prod_{i=1}^{p} \bar{t}_{i} \bar{z}^{-1}\right)^{-\tilde{a}_{0}}
\end{aligned}
$$

It is now obvious that the result of the integral for $|z|>1$ is deduced from the result for $|z|<1$ by a simple change of the parameters of the hypergeometric functions of argument $\frac{1}{z}$ up to the multiplicative factor

$$
(-1)^{s-\tilde{s}} z^{-a_{0}} \bar{z}^{-\tilde{a}_{0}}
$$

namely if we define $\alpha_{i}$ and $\beta_{i}$ as the parameters we get by simple identification

$$
\begin{aligned}
\alpha_{0} & =a_{0} \\
\alpha_{i} & =a_{0}-b_{i}+1 \\
\beta_{i} & =a_{0}-a_{i}+1
\end{aligned}
$$

which corresponds to the parameters of $W_{0}(z)$ and

$$
\begin{aligned}
\alpha_{i}-\beta_{j}+1 & =a_{j}-b_{i}+1 \\
\beta_{i}-\beta_{j}+1 & =a_{j}-a_{i}+1 \\
2-\beta_{j} & =a_{j}-a_{0}+1
\end{aligned}
$$

which corresponds to the parameters of $W_{j}(z)$. The above argument can be made rigorous using (14). 
To summarize

$$
I_{p+1}=\mu_{p+1} \sum_{j=0}^{p} \mu_{j} V_{j}(z) \tilde{V}_{j}(\bar{z})
$$

where

$$
\begin{gathered}
V_{j}(z)=z^{1-b_{j}}{ }_{p+1} G_{p}\left(a_{i}-b_{j}+1 ; b_{k}-b_{j}+1 ; z\right) \\
\mu_{p+1}=\frac{1}{\pi^{2}}(-1)^{a_{0}-\tilde{a}_{0}} \prod_{i=0}^{p} \Gamma\left(b_{i}-a_{i}\right) \Gamma\left(\tilde{b}_{i}-\tilde{a}_{i}\right) S\left(b_{i}-a_{i}\right)
\end{gathered}
$$

and

$$
\mu_{j}=\frac{\prod_{i=0}^{p} S\left(b_{j}-a_{i}\right)}{\prod_{i=0 \neq j}^{p} S\left(b_{j}-b_{i}\right)}
$$

and a similar formula for $|z|>1$.

\section{Acknowledgments}

The authors would like to thank R. Peschanski, M. Bauer and R. Guida for useful discussions. We would also like to thank $\mathrm{Ph}$ Di Fransesco for discussions leading to the use of Cauchy's Theorem in Lemma 4. JG would like to thank the Service Physique Theorique at Saclay for their hospitality during his visit especially P. Moussa. 


\section{Appendix A}

In this appendix we give an alternative method to calculate (30). Besides the conditions imposed in the hypothesis of Theorem (3) it will assumed that $b_{p}-\tilde{b}_{p}-b_{i}+\tilde{b}_{i} \geq 0$, $i=0, \ldots, p-1$. We need to calculate

$$
I=\sum_{i=0}^{p-1} \lambda_{i}^{p-1} \int t^{b_{p}-2} U_{i}(t) \bar{t}^{\tilde{b}_{p}-2} \tilde{U}_{i}(\bar{t}) d^{2} t
$$

where

$$
U_{i}(t)=t^{1-b_{i}} F_{p-1}\left(a_{j}-b_{i}+1 ; b_{k}-b_{i}+1 ; t\right)
$$

where $j, k \neq i=0, p-1$, and $\lambda_{i}^{p-1}$ is given in formula 11 and by convention $b_{0}=1$

We split the domain of integration in two parts, and write $I=I_{1}+I_{2}$ where $I_{1}$ is the contribution for $|t|<1$ and $I_{2}$ for $|t|>1$. In the first domain the hypergeometric functions are convergent so that

$$
I_{1}=\sum_{i=0}^{p-1} \lambda_{i}^{p-1} \iint_{B / \Gamma} t^{b_{p}-2} U_{i}(t) \tilde{t}^{\tilde{b}_{p}-2} \tilde{U}_{i}(\bar{t}) d^{2} t,
$$

where $B$ is the unit disk in the complex plane and $\Gamma$ is the contour used in the proof of Theorem 3. To calculate $I_{2}$, the other form of the solution which is valid for $|z|>1$ will be used, namely

$$
I_{2}=\sum_{i=0}^{p-1} \nu_{i}^{p-1} \int_{B^{c} / \Gamma} \int t^{b_{p}-2} W_{i}(t) \tilde{t}^{\tilde{b}_{p}-2} \tilde{W}_{i}(\bar{t}) d^{2} t
$$

where

$$
W_{i}(t)=t_{p}^{-a_{i}} F_{p-1}\left(a_{i}-b_{j}+1 ; a_{i}-a_{k}+1 ; \frac{1}{t}\right)
$$

with the same convention for the indices $j$ and $k$

By definition

$$
\begin{gathered}
\lambda_{i}^{p-1} \frac{\prod_{j=0 \neq i}^{p-1} \Gamma\left(b_{j}-b_{i}+1\right) \Gamma\left(\tilde{b}_{j}-\tilde{b}_{i}+1\right)}{\prod_{j=0}^{p-1} \Gamma\left(a_{j}-b_{i}+1\right) \Gamma\left(\tilde{a}_{j}-\tilde{b}_{i}+1\right)}=\mu_{p-1} \mu_{i}^{p-1} \\
\nu_{i}^{p-1} \frac{\prod_{j=0 \neq i}^{p-1} \Gamma\left(a_{i}-a_{j}+1\right) \Gamma\left(\tilde{a}_{i}-\tilde{a}_{j}+1\right)}{\prod_{j=0}^{p-1} \Gamma\left(a_{i}-b_{j}+1\right) \Gamma\left(\tilde{a}_{i}-\tilde{b}_{j}+1\right)}=(-1)^{s-\tilde{s}} \mu_{p-1} \rho_{i}^{p-1}
\end{gathered}
$$




$$
\begin{gathered}
s=\sum_{i=1}^{p-1} b_{i}-\sum_{i=0}^{p-1} a_{i} \\
\mu_{p-1}=(-1)^{a_{0}-\tilde{a}_{0}} \frac{1}{\pi^{2}} \prod_{i=0}^{p-1} \Gamma\left(b_{i}-a_{i}\right) \Gamma\left(\tilde{b}_{i}-\tilde{a}_{i}\right) S\left(b_{i}-a_{i}\right) \\
\mu_{i}^{p-1}=\frac{\prod_{j=0}^{p-1} S\left(b_{i}-a_{j}\right)}{\prod_{j=0 \neq i}^{p-1} S\left(b_{i}-b_{j}\right)}
\end{gathered}
$$

and

$$
\rho_{i}^{p-1}=\frac{\prod_{j=0}^{p-1} S\left(b_{j}-a_{i}\right)}{\prod_{j=0 \neq i}^{p-1} S\left(a_{j}-a_{i}\right)}
$$

For $w$ real and $|w| \leq r<1$ set,

$$
I_{1}^{i}(w)=\iint_{B / \Gamma}(w t)^{b_{p}-2} U_{i}(w t)(\overline{w t})^{\tilde{b}_{p}-2} \tilde{U}_{i}(\overline{w t}) d^{2} t
$$

and expand $U_{i}(t)\left(\operatorname{resp} \tilde{U}_{i}(\bar{t})\right)$ as a series $\sum_{n}\left(\operatorname{resp} \sum_{m}\right)$. The integration on the azimuthal angle $\phi$ yields at once

$$
m=n+b_{p}-\tilde{b}_{p}-b_{i}+\tilde{b}_{i}
$$

The integral over the modulus of $t$ is also straightforward and yields an additional factor

$$
\frac{1}{2} \frac{\Gamma\left(n+b_{p}-b_{i}\right)}{\Gamma\left(n+b_{p}-b_{i}+1\right)}\left(w^{2}\right)^{n+b_{p}-b_{i}}
$$

Collecting all the pieces we get

$$
I_{1}^{i}(w)=\pi \mu_{p-1} \mu_{i}^{p-1} w^{2\left(b_{p}-b_{i}-1\right)}{ }_{2 p+1} G_{2 p}^{i}\left(w^{2}\right)
$$

where ${ }_{2 p+1} G_{2 p}^{i}\left(w^{2}\right)$ is the unrenormalized hyper geometric function the upper parameters of which are $a_{j}-b_{i}+1, \tilde{a}_{j}-b_{i}+1+b_{p}-\tilde{b}_{p}, b_{p}-b_{i}$ and the lower ones read $b_{k}-b_{i}+1, \tilde{b}_{k}-b_{i}+1+b_{p}-\tilde{b}_{p}, b_{p}-b_{i}+1,1+b_{p}-\tilde{b}_{p}-b_{i}+\tilde{b}_{i}$.

Now define

$$
f_{R}(w)=\pi^{2} \mu_{p-1} \frac{1}{2 i \pi} \int_{C_{R}} d t \prod_{j=0}^{p-1} \frac{\Gamma\left(1-b_{j}-t\right) \Gamma\left(b_{p}-\tilde{b}_{p}+\tilde{a}_{j}+t\right)}{\Gamma\left(1-a_{j}-t\right) \Gamma\left(b_{p}-\tilde{b}_{p}+\tilde{b}_{j}+t\right)} \frac{\Gamma\left(t+b_{p}-1\right)}{\Gamma\left(t+b_{p}\right)} w^{2\left(b_{p}-1+t\right)}
$$


where $C_{R}$ is a loop beginning and ending at $+\infty$ and encircling all poles of $\Gamma(\ldots-t)$ once in the negative direction but none of the poles of $\Gamma(\ldots+t)$. This yields at once

$$
I_{1}(w)=f_{R}(w)
$$

The same method holds for $I_{2}(w), w$ real and $|w| \geq r>1$ with the integration over the azimuthal angle yielding $n=m+b_{p}-\tilde{b}_{p}-a_{i}+\tilde{a}_{i}$ while the integration on the modulus of $t$ gives

$$
\frac{1}{2} \frac{\Gamma\left(\tilde{a}_{i}-\tilde{b}_{p}+1+m\right)}{\Gamma\left(\tilde{a}_{i}-\tilde{b}_{p}+2+m\right)}\left(w^{2}\right)^{-m-1}+b_{\tilde{p}}-a_{\tilde{i}} .
$$

As before $I_{2}(w)$ is a complicated sum of $G$ functions.

Now define,

$$
\begin{aligned}
f_{L}(w)= & (-1)^{s-\tilde{s}} \pi^{2} \mu_{p-1} \frac{1}{2 i \pi} \int_{C_{l}} d t \\
& \prod_{j=0}^{p-1} \frac{\Gamma\left(1-b_{j}-t\right) \Gamma\left(b_{p}-\tilde{b}_{p}+\tilde{a}_{j}+t\right)}{\Gamma\left(1-a_{j}-t\right) \Gamma\left(b_{p}-\tilde{b}_{p}+\tilde{b}_{j}+t\right)} \frac{\Gamma\left(t+b_{p}-1\right)}{\Gamma\left(t+b_{p}\right)} w^{2\left(b_{p}-1+t\right),},
\end{aligned}
$$

where $C_{L}$ is a loop beginning and ending at $-\infty$ and encircling all poles of $\Gamma(\ldots+t)$ once in the positive direction but none of the poles of $\Gamma(\ldots-t)$.

It is easy to check that

$$
f_{L}=-I_{2}(w)+\text { residus at } t=1-b_{p} .
$$

Indeed

$$
\rho_{i}^{p-1}=(-1)^{s-\tilde{s}} \frac{\prod_{j=0}^{p-1} S\left(\tilde{b}_{j}-\tilde{a}_{i}\right)}{\prod_{j=0 \neq i}^{p-1} S\left(\tilde{a}_{j}-\tilde{a}_{i}\right)} .
$$

But Cauchy theorem implies that

$$
f_{L}(w)=f_{R}(w),
$$

so that in the limit $w \rightarrow 1$ we find,

$$
I=\pi^{2} \mu_{p-1} \prod_{j=0}^{p-1} \frac{\Gamma\left(b_{p}-b_{j}\right) \Gamma\left(1-\tilde{b}_{p}+\tilde{a}_{j}\right)}{\Gamma\left(b_{p}-a_{j}\right) \Gamma\left(1-\tilde{b}_{p}+\tilde{b}_{j}\right)}
$$


or equivalently

$$
I=\mu_{p-1} \prod_{j=0}^{p-1} \frac{S\left(b_{p}-a_{j}\right)}{S\left(b_{p}-b_{j}\right)} \prod_{j=0}^{p-1} \frac{\Gamma\left(1+a_{j}-b_{p}\right) \Gamma\left(1-\tilde{b}_{p}+\tilde{a}_{j}\right)}{\Gamma\left(1-b_{p}+b_{j}\right) \Gamma\left(1+\tilde{b}_{j}-\tilde{b}_{p}\right)}
$$

At this stage it is worth noticing that

$$
\mu_{p}=\mu_{p-1} \Gamma\left(b_{p}-a_{p}\right) \Gamma\left(\tilde{b}_{p}-\tilde{a}_{p}\right) S\left(b_{p}-a_{p}\right),
$$

which can be recast as,

$$
(-1)^{b_{p}-\tilde{b}_{p}-\left(a_{p}-\tilde{a}_{p}\right)} \mu_{p-1} \pi^{2}=\frac{\mu_{p}}{\Gamma\left(1+a_{p}-b_{p}\right) \Gamma\left(1+\tilde{a}_{p}-\tilde{b}_{p}\right) S\left(b_{p}-a_{p}\right)} .
$$

this yields at once

$$
I=(-1)^{b_{p}-\tilde{b}_{p}-\left(a_{p}-\tilde{a}_{p}\right)} \lambda_{p+1}^{p}
$$

This is the expected result.

As a nice application we may recover the generalized Euler formula, namely calculate

$$
I=\int t^{a-1} U_{0}(t) \bar{t}^{\tilde{a}-1} \tilde{U}_{0}(\bar{t}) d^{2} t
$$

where $U_{0}(t)=(1-t)^{b-1}={ }_{1} F_{0}(b-1, t)$. If we follow the method presented above we are led to calculate

$$
f_{R}=\frac{1}{2 i \pi} \int_{C_{R}} d t \frac{\Gamma(-t) \Gamma(a-\tilde{a}+1-\tilde{b}+t)}{\Gamma(b-t) \Gamma(a-\tilde{a}+1+t)} \frac{\Gamma(t+a)}{\Gamma(t+a+1)}
$$

since by identification $b_{1}=a-1, a_{0}=1-b$, it is easy to get that

$$
f_{R}=I S(b) \Gamma(1-b) \Gamma(1-\tilde{b}) \pi^{2}
$$

by picking the pole at $t=-a$, we get at once

$$
f_{L}=\frac{\Gamma(a) \Gamma(1-\tilde{a}-\tilde{b})}{\Gamma(b+a) \Gamma(1-\tilde{a})}
$$

collecting all the pieces the final result reads

$$
I=\pi \frac{\Gamma(a) \Gamma(b) \Gamma(1-\tilde{a}-\tilde{b})}{\Gamma(1-\tilde{b}) \Gamma(b+a) \Gamma(1-\tilde{a})}
$$




\section{References}

[1] L.N Lipatov, Sov. J. Nucl. Phys. 23 (1976) 642; V.S. Fadin, E.A. Kuraev and L.N. Lipatov, Phys. Lett B 60 (1975) 50; E.A. Kuraev, L.N. Lipatov and V.S. Fadin, Sov. Phys. JETP 44 (1976) 45, 45 (1977) 199; I.I. Balitsky and L.N. Lipatov, Sov. J. Nucl. Phys. 28 (1978) 822; L.N. Lipatov zh. Eksp. Teor. FizRto . 90 (1986) 1536 (Eng. Trans. Sov. Phys. JETP 63 (1986) 904.

[2] H. Navelet and R. Peschanski Nucl. Phys. B 507 (1997) 353-366.

[3] See for instance A. Bialas, H. Navelet and R. Peschanski, The QCD triple Pomeron coupling from string amplitudes, Saclay Preprint T97/145, hepph/9711442. G.P. Korchemsky, Conformal bootstrap for the BFKL Pomeron, Orsay Preprint LPTHE 97-62 and hep-ph/9711277.

[4] A.M. Polyakov, Zh. Eksp. Teor. Fiz. Lett. 12 (1970) 538, 66 (1974) 23; A.A. Migdal, Phys. Lett B44 (1972) 112; A.A. Belavin, A.M. Polyakov and A.B. Zamolodchikov, Nucl. Phys. B 241 (1984) 333; Vl.S. Dotsenko and V.A. Fateev Nucl. Phys B 240 FS12 (1994) 312. For a collection of reprints on conformal invariance: see Conformal invariance and applications to statistical mechanics, C. Itzykson, H. Saleur, J.B. Zuber eds., World Scientific (1988). Conformal field theory, Ph. Di Francesco, P. Mathieu, D. Senechal Springer (1997). R. Guida and N. Magnoli to be published in International J. Phys. A (see appendix A and B). S.D. Mathur, Nucl. Phys B 369 (1992).

[5] R. Peschanski Phys. Lett. B 409 (1997) 49. A.H. Mueller, B. Patel, Nucl. Phys. B 425 (1994) 471. M.A. Braun and G.P Vacca Bologna university preprint, hep-ph/9711486. J. Bartels Zeit.für Phys. C 60 (1993) 471.

[6] Higher Transcendental Functions, Vol. 1, A. Erdelyi ed., McGraw-Hill, 1953 NY, formula (33), p. 12.

[7] G. H. Watson, A Treatise on the Theory of Bessel Functions, Cambridge (1995).

[8] A. P. Prudnikov,Yu. A. Brychkov, O. I.Marichev, Integral and Series, Vol 3 Gordon and Breach, NY, 1990

[9] E. A. Coddington and N. Levinson Theory of Ordinary Differential Equations McGraw-Hill, NY (1955). 
[10] Y. L. Luke Special Functions and Their Applications Academic Press, NY (1969) Vol. 1. 